\title{
Stability Augmentation and Active Flutter Suppression of a Flexible Flying-Wing Drone
}

\author{
Dr. David K. Schmidt ${ }^{1}$ \\ University of Colorado \\ Monument, Co 80132
}

\begin{abstract}
Integrated control laws are developed for stability augmentation and active flutter suppression (AFS) of a flexible, flying-wing drone. The vehicle is a 12-pound unmanned, flyingwing research aircraft with a $10 \mathrm{ft}$ wingspan. AFS is flight critical since the subject vehicle is designed to flutter within its flight envelope. The critical flutter condition involves aeroelastic interactions between the rigid-body and elastic degrees of freedom; hence the control laws must simultaneously address both rigid-body stability augmentation and flutter suppression. The control-synthesis approach is motivated by the concept of Identically Located Force and Acceleration (ILAF), successfully applied on some previous operational aircraft. Based on the flutter characteristics and on conventional stability-augmentation concepts, two simple loop closures are suggested. It is shown that this control architecture robustly stabilizes the bodyfreedom-flutter condition, increases the damping of the second aeroelastic mode (which becomes a second flutter mode at higher velocity), and provides reasonably conventional vehicle pitchattitude response. The critical factors limiting the performance of the feedback system are identified to be the bandwidth of the surface actuators and the pitch effectiveness of the control surfaces.
\end{abstract}

\section{Introduction}

We seek to develop integrated control laws for stability augmentation and active flutter suppression (AFS) for a flexible, flying-wing research drone. AFS is flight critical in this instance

\footnotetext{
${ }^{1}$ Professor Emeritus, Fellow, AIAA.

Copyright (C) 2015 by David K. Schmidt. Permission to duplicate and distribute is hereby granted to AIAA.
} 
since the subject vehicle is designed to flutter within the flight envelope. The vehicle of interest, depicted in Figs. 1 and 2, is Lockheed Martin's Free-Flying Aeroelastic Demonstrator (FFAD), a 12-pound unmanned, flying-wing aircraft with a $10 \mathrm{ft}$ wingspan (Ref. 1), The vehicle is a lowspeed swept-back flying wing with winglets on the wing tips for directional stability, and an electric motor driving a pusher propeller (not shown in the figure) mounted at the top rear of the rigid center body. The entire trailing edge of the wing consists of eight control surfaces. The planform parameters are listed in Table 2, and the mass properties are given in Table 3, all from Ref. 2. Note that this is a fairly small, lightweight, unmanned vehicle.

One of these vehicles was provided for flight research to the Unmanned Air Vehicle (UAV) Lab of the University of Minnesota (UMN), and we are collaborating in this research. These vehicles were precursors to the unmanned, multi-utility X-56A vehicle currently undergoing flight testing at NASA's Armstrong Flight Research Center.

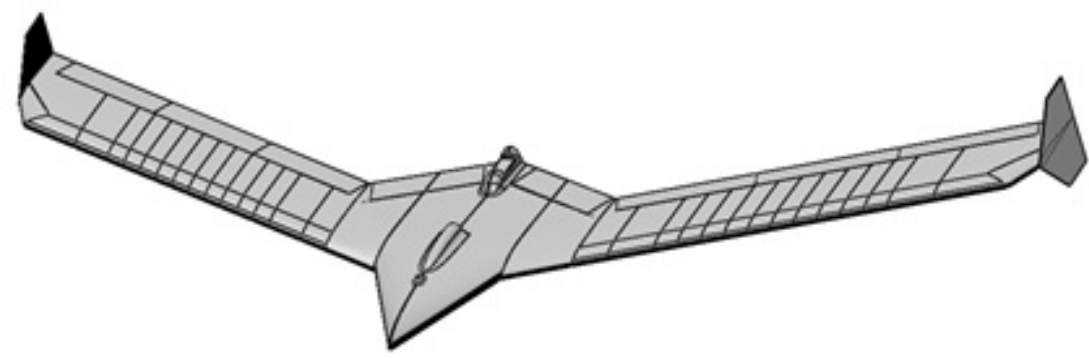

Figure 1, Vehicle Configuration

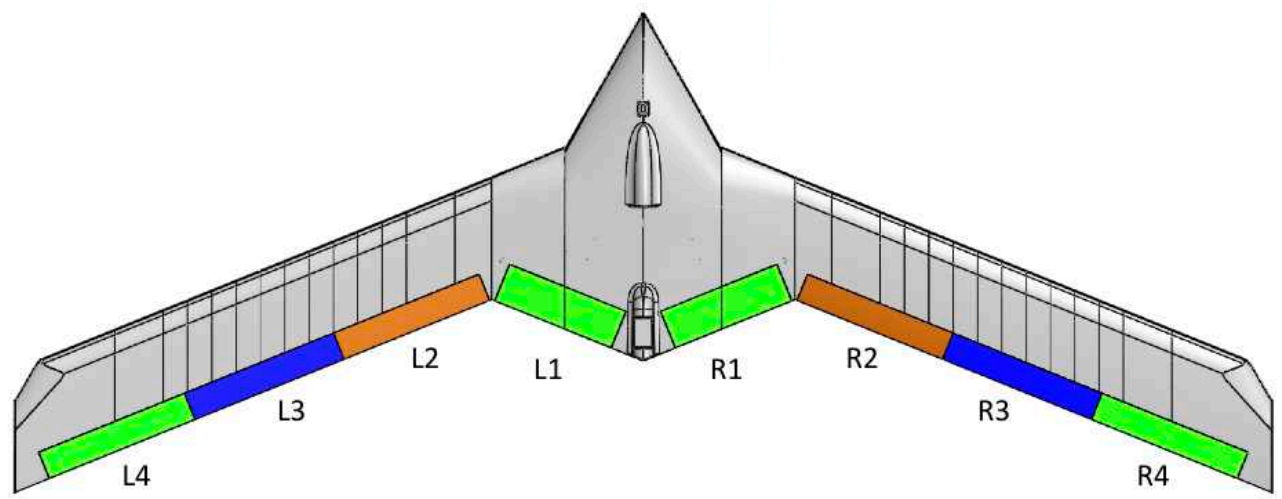

Figure 2, Vehicle Planform View 
Table 1, Planform Description

\begin{tabular}{|c|c|c|c|c|c|}
\hline $\begin{array}{c}\text { Planform } \\
\text { Area, } \mathbf{S}\end{array}$ & Span, $\boldsymbol{b}$ & $\begin{array}{c}\text { Aspect } \\
\text { Ratio, } \boldsymbol{A} \boldsymbol{R}\end{array}$ & $\begin{array}{c}\text { Taper } \\
\text { Ratio, } \boldsymbol{\lambda}\end{array}$ & M.A.C., $\bar{c}$ & LE Sweep, $\boldsymbol{\Lambda}_{\mathbf{L E}}$ \\
\hline $11.67 \mathrm{ft}^{2}$ & $10 \mathrm{ft}$ & 8.57 & 4.25 & $1.313 \mathrm{ft}$ & $22 \mathrm{deg}$ \\
\hline
\end{tabular}

Table 2, Vehicle Mass Properties

\begin{tabular}{|l|l|}
\hline Property & Value \\
\hline Total Weight & $1.99 \mathrm{lb}$ \\
\hline C.G. Location & $23.26 \mathrm{in}$ (from nose) \\
\hline Pitch Moment of Inertia & $1245.8 \mathrm{lb}-\mathrm{in}^{2}$ \\
\hline Roll Moment of Inertia & $8529.5 \mathrm{lb}-\mathrm{in}^{2}$ \\
\hline Yaw Moment of Inertia & $8118.4 \mathrm{lb}-\mathrm{in}^{2}$ \\
\hline Product of Inertia (est) & $-0.30 \mathrm{ln}-\mathrm{in}^{2}$ \\
\hline
\end{tabular}

\section{The NDOF Dynamic Model}

A previously developed n-degree-of-freedom (NDOF) math model of the longitudinal dynamics (Ref. 3) will be utilized in the control-law synthesis. And a review of this model development and the vehicle dynamics will significantly aid in understanding the control-law development. The modeling approach yields an integrated NDOF model based on first principles, derived in the vehicle-fixed, or non-inertial, reference frame. The results in Ref. 3 were compared to those of Lockheed Martin (Ref. 1), and good agreement was indicated. Although the vehicle is statically stable in pitch, it is predicted to exhibit both body-freedom and bending torsion flutter at 47 and $57 \mathrm{kt}$, respectively.

Only the first three symmetric free vibration modes were used in the development of the NDOF dynamic model, but these were shown to be sufficient to capture the critical aspects of the dynamics. The modal data for these three vibration modes are given in Table 3, along with the data sources. The values from the UMN GVTs highlighted in bold were used in the dynamic modeling. 
Table 3, Structural Vibration Characteristics

\begin{tabular}{|c|c|c|c|}
\hline Data and Source & $\mathbf{1}^{\text {st }}$ Sym Mode & $\mathbf{2}^{\text {nd }}$ Sym Mode & $\mathbf{3}^{\text {rd }}$ Sym Mode \\
\hline Frequency, UMN FEM & $34.9 \mathrm{r} / \mathrm{s}$ & $94.5 \mathrm{r} / \mathrm{s}$ & $163.2 \mathrm{r} / \mathrm{s}$ \\
\hline Frequency, UMN GVT & $\mathbf{3 4 . 6} \mathbf{r} / \mathbf{s}$ & $\mathbf{1 1 7 . 8} \mathbf{~ r / s}$ & $\mathbf{1 4 5 . 6} \mathbf{r} / \mathbf{s}$ \\
\hline Frequency, LM GVT & $35.4 \mathrm{r} / \mathrm{s}$ & $123.4 \mathrm{r} / \mathrm{s}$ & $147.3 \mathrm{r} / \mathrm{s}$ \\
\hline Damping, UMN GVT & $1.55 \%$ & $2.06 \%$ & $2.85 \%$ \\
\hline Gen. Mass, UMN FEM & $0.28950 \mathrm{~s} 1-\mathrm{ft}^{2}$ & $0.00772 \mathrm{sl}-\mathrm{ft}^{2}$ & $0.05239 \mathrm{sl- \textrm {ft } ^ { 2 }}$ \\
\hline
\end{tabular}

The mode shapes for these three symmetric modes, obtained from the FEM, are shown in Figs. 3-5, respectively. Note that in these modes vertical $(Z)$ translations are positive down, and wing-torsional displacements $\theta$ are positive leading-edge up. It is clear from Figs. 3 and 5 that the first and third symmetric modes exhibit both bending and torsional displacements, but are referred to herein as "bending" modes. The second symmetric mode shape in Fig. 4 exhibits almost pure torsional displacement.

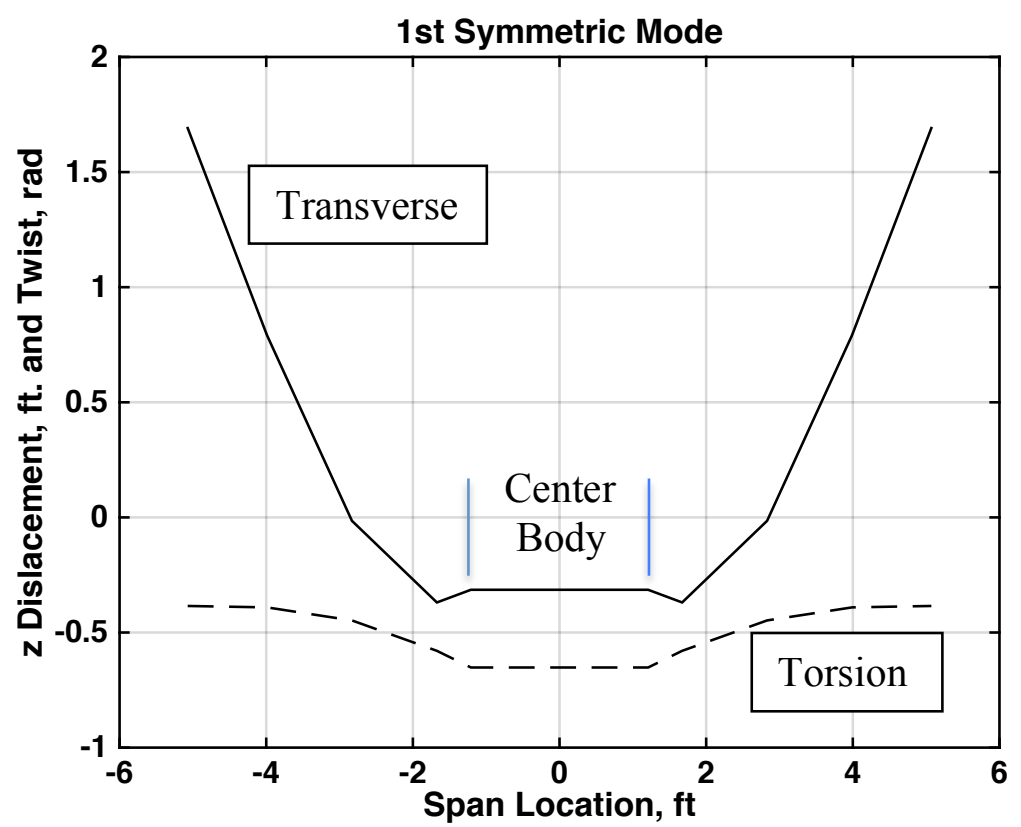

Figure 3, Mode Shape, First Symmetric Mode 


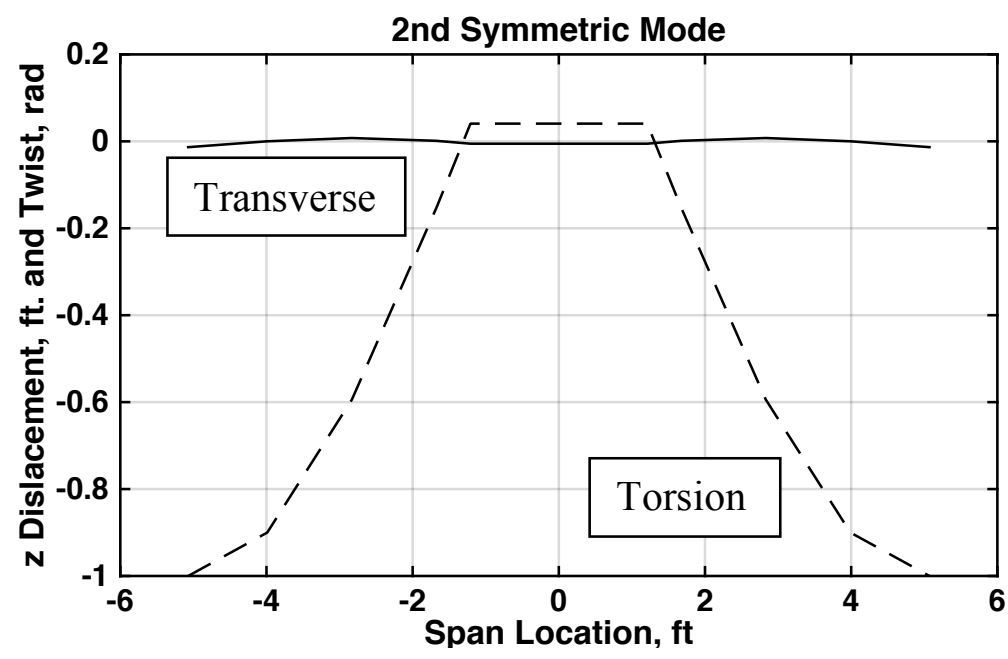

Figure 4, Mode Shape, Second Symmetric Mode

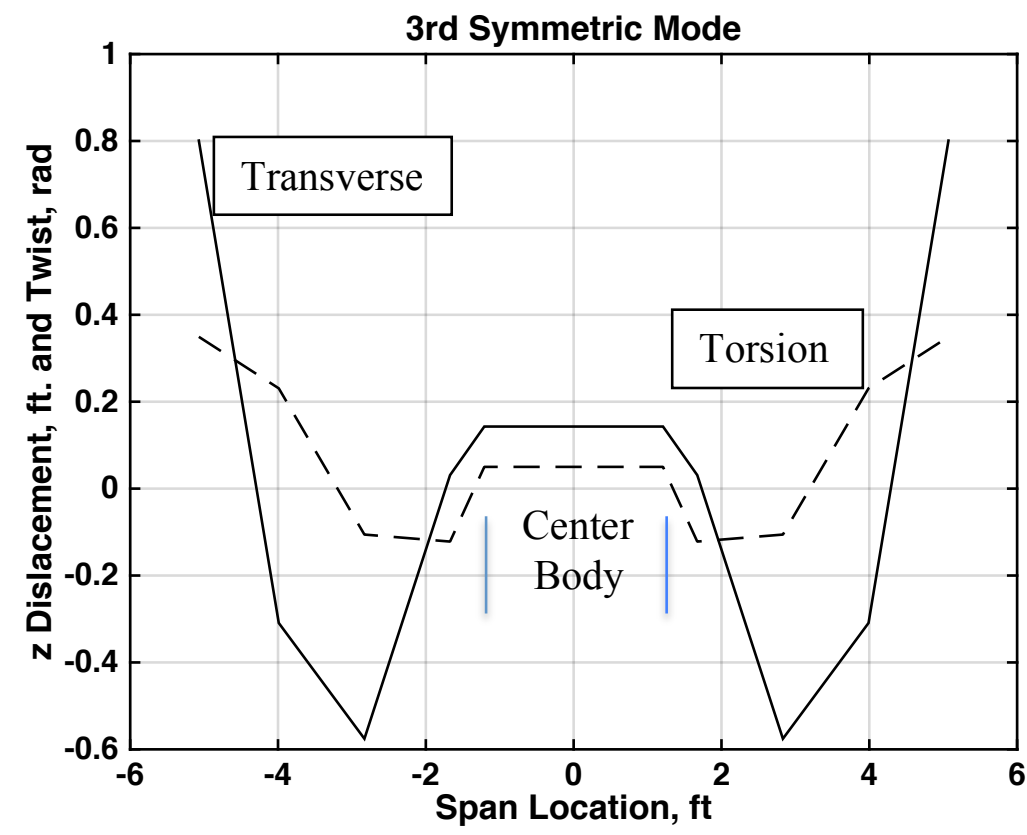

Figure 5, Mode Shape, Third Symmetric Mode

We will revisit these mode shapes again many times. But note here that the first mode is characterized by nose-down pitch/torsion in phase with wing-tip-down transverse displacement. Plus the bending and torsion in the first and third modes involves displacements of the vehicle center body as well as the wings.

As noted in Ref. 4, one can mathematically describe the instantaneous shape of the structure in terms of free-vibration mode shapes and modal coordinates. That is, given sufficiently 
large $n$, the instantaneous elastic deformation $\mathbf{d}_{E}$ of the vehicle's structure at some location $\mathbf{p}$ on the undeformed vehicle can be expressed in terms of the $n$ vibration mode shapes evaluated at location $\mathbf{p}$, or $\mathbf{v}_{i}(\mathbf{p})$ and the $n$ time-dependent vibration modal coordinates $\eta_{i}(t)$, or

$$
\mathbf{d}_{E}(\mathbf{p}, t)=\sum_{i=1}^{n} \mathbf{v}_{i}(\mathbf{p}) \eta_{i}(t)
$$

When all $\eta_{i}=0$, therefore, the vehicle is in its undeformed shape.

The system states in the NDOF dynamic model associated with the $n$ elastic degrees of freedom are chosen as these vibration modal coordinates $\eta_{i}(t)$. Furthermore, from Lagrange's equation, these $n$ modal coordinates are governed by $n$ second order differential equations of the following form

$$
\ddot{\eta}_{i}(t)+2 \zeta_{i} \omega_{i} \dot{\eta}_{i}(t)+\omega_{i}^{2} \eta_{i}(t)=Q_{i} / m_{i}
$$

where

$$
\begin{aligned}
& \zeta_{i}=\text { vibration modal damping } \\
& \omega_{i}=\text { in-vacuo vibration modal frequency } \\
& Q_{i}=\text { generalized force acting on the i'th modal coordinate } \\
& m_{i}=\text { generalized mass of the } i \text { 'th vibration mode }
\end{aligned}
$$

The NDOF dynamic model used includes the first three symmetric vibration modes, so here $n=3$.

The linear NDOF model for the longitudinal dynamics takes the form given in Eqns. 3 below, with the first four states corresponding to the three rigid-body degrees of freedom, while the last six states correspond to the three elastic degrees of freedom. In the absence of unsteadyaerodynamic effects, there are no additional states associated with lagged aerodynamic. Also, this model is for the "bare airframe" only. Actuator dynamics are added later. Note the natural partitioning of the system into inertially decoupled rigid-body and elastic subsystems. The terms 
$Z_{\eta}, M_{\eta}$, and $\Xi$. are the dimensional aeroelastic stability derivatives, which capture the aerodynamic coupling between all the degrees of freedom. These derivatives are functions of flight condition, mass properties (including generalized masses), and the vibration mode shapes discussed above.

$$
\begin{aligned}
& \mathbf{x}^{T}=\left[\begin{array}{llll:llllll}
u_{r i g} & \alpha_{r i g} & \theta_{r i g} & q_{r i g} & \eta_{1} & \dot{\eta}_{1} & \eta_{2} & \dot{\eta}_{2} & \eta_{3} & \dot{\eta}_{3}
\end{array}\right] \\
& \mathbf{A}=\left[\begin{array}{ccccc:cccc}
X_{u} & X_{\alpha} & -g & X_{q} & 0 & 0 & \cdots & 0 & 0 \\
Z_{u} / \mathrm{U}_{0} & Z_{\alpha} / \mathrm{U}_{0} & 0 & 1+Z_{q} / \mathrm{U}_{0} & Z_{\eta_{1}} / \mathrm{U}_{0} & Z_{\dot{\eta}_{1}} / \mathrm{U}_{0} & \cdots & Z_{\eta_{3}} / \mathrm{U}_{0} & Z_{\dot{\eta}_{3}} / \mathrm{U}_{0} \\
0 & 0 & 0 & 1 & 0 & 0 & \cdots & 0 & 0 \\
M_{u} & M_{\alpha} & 0 & M_{q} & M_{\eta_{1}} & M_{\dot{\eta}_{1}} & \cdots & M_{\eta_{3}} & M_{\dot{\eta}_{3}} \\
\hdashline 0 & 0 & 0 & 0 & 0 & 1 & \cdots & 0 & 0 \\
0 & \Xi_{1_{\alpha}} & 0 & \Xi_{1_{q}} & \Xi_{1_{\eta_{1}}}-\omega_{1}^{2} & \Xi_{\eta_{\eta_{1}}}-2 \zeta_{1} \omega_{1} & \cdots & \Xi_{\eta_{\eta_{3}}} & \Xi_{\eta_{\eta_{3}}} \\
\vdots & \vdots & \vdots & \vdots & \vdots & \vdots & \cdots & \vdots & \vdots \\
0 & 0 & 0 & 0 & 0 & 0 & \cdots & 0 & 1 \\
0 & \Xi_{3_{\alpha}} & 0 & \Xi_{3_{q}} & \Xi_{0_{\eta_{1}}} & \Xi_{3_{\eta_{1}}} & \cdots & \Xi_{\eta_{\eta_{3}}}-\omega_{3}^{2} & \Xi_{3_{\eta_{3}}}-2 \zeta_{3} \omega_{3}
\end{array}\right] \\
& \mathbf{A}=\left[\begin{array}{ccc}
\mathbf{A}_{R R} & \mathbf{A}_{R E} \\
\hdashline \mathbf{A}_{E R} & \mathbf{A}_{E E}
\end{array}\right] \quad \mathbf{B}=\left[\begin{array}{ccc}
X_{\delta_{1}} & \cdots & X_{\delta_{4}} \\
Z_{\delta_{1}} / \mathrm{U}_{0} & \cdots & Z_{\delta_{4}} / \mathrm{U}_{0} \\
0 & \cdots & 0 \\
M_{\delta_{1}} & \cdots & M_{\delta_{4}} \\
\hdashline 0 & \cdots & 0 \\
\Xi_{\delta_{1}} & \cdots & \Xi_{\delta_{\delta_{4}}} \\
0 & \cdots & 0 \\
\Xi_{\delta_{\delta_{1}}} & \cdots & \Xi_{\delta_{\delta_{4}}} \\
0 & \cdots & 0 \\
\Xi_{\delta_{\delta_{4}}} & \cdots & \Xi_{3_{\delta_{4}}}
\end{array}\right]=\left[\begin{array}{c}
\mathbf{B}_{R} \\
\hdashline \mathbf{B}_{E}
\end{array}\right] \quad 3
\end{aligned}
$$

\section{The Flutter Characteristics}

Now consider Fig. 6, showing the dynamic-pressure root locus for the model just described. The eigenvalues of the system (A) matrix are shown for seven flight velocities between 30 and $60 \mathrm{kt}$. The system modes are labeled according to their modal genesis. That is, the mode 
branches are identified according to their genesis mode of pure rigid-body mode or pure freevibration mode with no aerodynamic forces. But to be clear, all the modes involve coupling through the aerodynamics, and hence are not pure short period, pure vibration, etc.

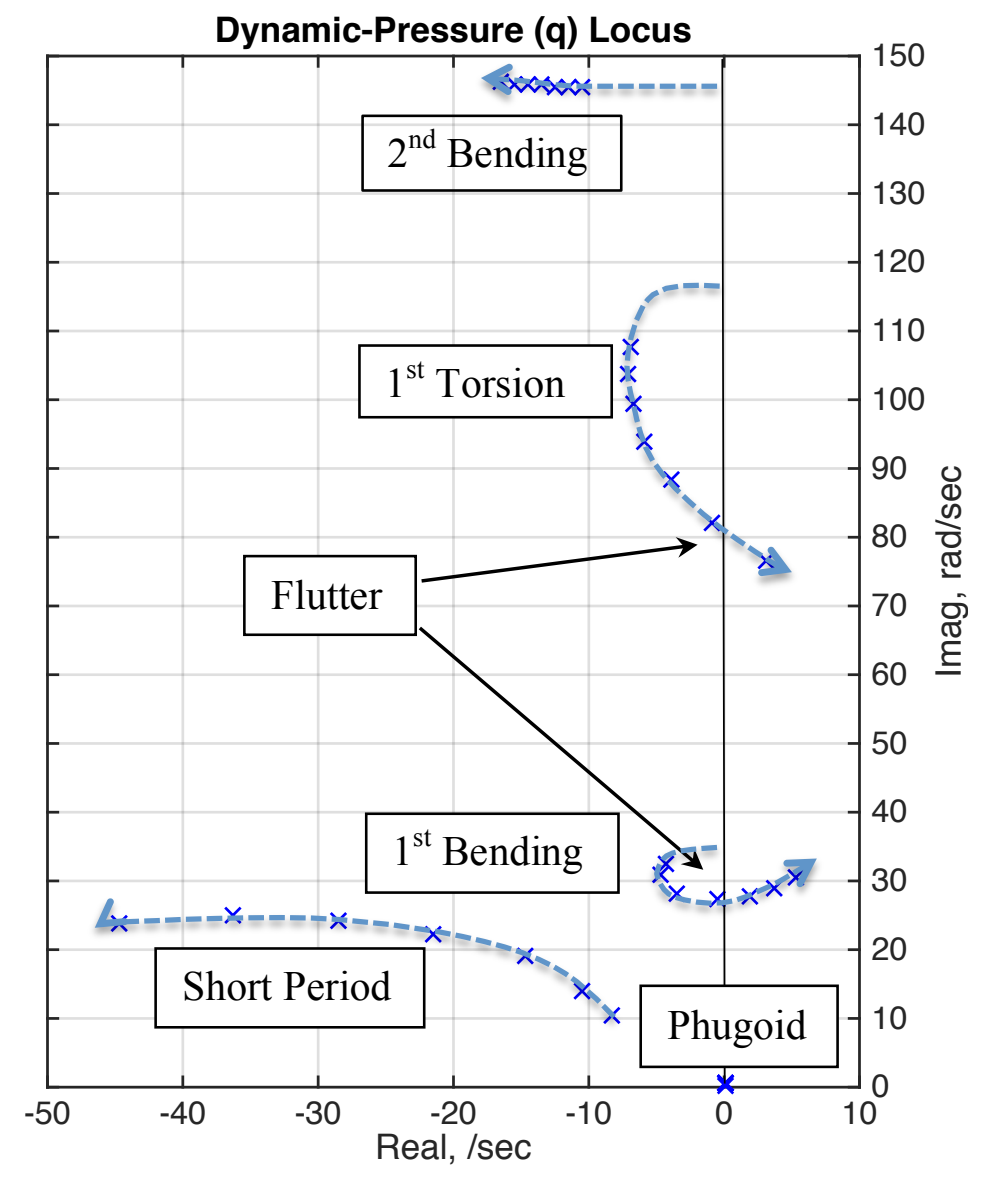

Figure 6, Dynamic-Pressure Root Locus

Next consider the branch labeled "Short Period." As the flight velocity, or dynamic pressure, increases, the natural frequency and damping of this mode both increase. The next branch labeled " $1{ }^{\text {st }}$ Bending" begins at the pole from the first symmetric-bending vibration mode, almost on the imaginary axis at $34.6 \mathrm{rad} / \mathrm{sec}$, and as flight velocity increases this branch eventually crosses the imaginary axis. This axis crossing corresponds to the BFF condition, which here occurs at a flight velocity of approximately $47 \mathrm{kt}$ and with a frequency of $27.5 \mathrm{rad} / \mathrm{sec}(4.4$ $\mathrm{Hz})$. 
Next, with regard to the branch labeled " 1 st Torsion," the branch begins at the pole arising from the second symmetric vibration mode (torsion mode), almost on the imaginary axis at 117.8 $\mathrm{rad} / \mathrm{sec}$, and at higher flight velocity it also crosses the imaginary axis, indicating a second flutter mode. This flutter condition is predicted to occur at a flight velocity of $57 \mathrm{kt}$, and with a frequency of $80 \mathrm{rad} / \mathrm{sec}(12.7 \mathrm{~Hz})$.

Finally, consider the branch labeled " 2 nd $B$ ending. This branch begins near the imaginary axis at $145.6 \mathrm{rad} / \mathrm{sec}$, this mode's vibration frequency, and as the flight velocity increases, these roots simply move further into the left half plane. The Phugoid roots remain near the origin at all flight velocities.

Some variations on the above model were also considered in Ref. 3, which involve truncating or residualizing the second and third elastic degrees of freedom. Residualization yields a reduced-order model that retains the static-elastic effects of the residualized degrees of freedom on the aerodynamics, while truncation eliminates both the dynamics and the static-elastic aerodynamic effects of the truncated degrees of freedom.

Truncation or residualization of the second and third elastic degrees of freedom leads to quite different results. With a truncated model neither the "Short-Period" nor the " 1 st Bending" roots move as much with flight velocity, and no flutter instability is indicated over this range of velocity. So the "Short-Period" and "1st-bending" degrees of freedom interact much less in the absence of the " $1^{\text {st }}$ Torsion" mode. In contrast, the dynamics of the residualized model are quite similar to those for the full-order model. There exists considerable interaction between the "ShortPeriod" and " 1 st Bending" modes in this case, and such a model also suggests BFF occurs at a flight velocity of approximately $47 \mathrm{kt}$ with a frequency of $27.5 \mathrm{rad} / \mathrm{sec}$. These results are almost identical to those obtained from the full-order model for the BFF condition, and these combined 
results also indicate that the static-elastic effects of the first-torsion mode, which are included in a residualized model, are important to the existence of BFF.

When comparing these results from Ref. 3 with LM's results reported in Ref. 1, quite good agreement was found in terms of the presence of both flutter conditions, and with regards to the flutter speeds, flutter frequencies, and genesis modes of the flutter conditions. Plus, the flighttest results reported in Ref. 1 confirmed a critical flutter speed of approximately $46 \mathrm{kts}$. All these results are summarized in Table 6 below.

Table 6, Comparison Of Flutter Speeds and Frequencies

\begin{tabular}{|c|c|c|c|c|}
\hline Model/Test & $\begin{array}{c}\text { BFF Flutter } \\
\text { Speed }\end{array}$ & $\begin{array}{c}\text { BFF Flutter } \\
\text { Frequency }\end{array}$ & $\begin{array}{c}\text { BT Flutter } \\
\text { Speed }\end{array}$ & $\begin{array}{c}\text { BT Flutter } \\
\text { Frequency }\end{array}$ \\
\hline LM Analytical & $43 \mathrm{kt}$ & $4.2 \mathrm{~Hz}$ & $57 \mathrm{kt}$ & $10.5 \mathrm{~Hz}$ \\
\hline LM Flight Test & $46 \mathrm{kt}$ & $4.5 \mathrm{~Hz}$ & $\mathrm{NA}$ & $\mathrm{NA}$ \\
\hline Full-Order Model & $47 \mathrm{kt}$ & $4.4 \mathrm{~Hz}$ & $57 \mathrm{kt}$ & $12.7 \mathrm{~Hz}$ \\
\hline Residualized Model & $47 \mathrm{kt}$ & $4.4 \mathrm{HZ}$ & $\mathrm{NA}$ & $\mathrm{NA}$ \\
\hline Truncated Model & No Flutter & No Flutter & NA & NA \\
\hline
\end{tabular}

\section{Attitude Dynamics}

The transfer functions for pitch-attitude and plunge-acceleration (measured at the $c g$ location of the undeformed vehicle) from elevator $\left(\delta_{3}\right)$ are given below. (Here a shorthand notation in used. Two terms in square brackets denote the damping and frequency of a quadratic polynomial, and a single term in brackets denotes the negative of the root of a first-order polynomial.) The flight condition is $3000 \mathrm{ft}$ altitude and $60 \mathrm{fps}$ velocity, below the critical flutter speed.

$$
\begin{aligned}
& \frac{\theta_{c g}(s)}{-\delta_{3}(s)}=\frac{65.24[0.0536][7.044][0.22,41.2][0.05,101.7][0.05,165.3]}{[-0.01,0.61][0.59,18.1][0.15,30.9][0.07,103.7][0.08,146.0]} \mathrm{deg} / \mathrm{deg} \\
& \frac{n_{Z-c g}(s)}{-\delta_{3}(s)}=\frac{-0.2276[0][-0.0279][29.58][-25.58][0.24,42.3][0.07,104.1][-282.6][246]}{[-0.01,0.61][0.59,18.1][0.15,30.9][0.07,103.7][0.08,146.0]} \mathrm{ft} / \mathrm{sec}^{2} / \mathrm{deg}
\end{aligned}
$$


The lowest frequency mode is the Phugoid, while the next higher-frequency is a shortperiod-like mode, discussed further below. The presence of the three aeroelastic modes is indicated by the three sets of higher-frequency dipoles (pole-zero pairs) in each of these transfer functions. The two "highest-frequency" zeros in the acceleration transfer function are effectively at infinity in the complex plane.

The second mode, with a natural frequency of around $18 \mathrm{rad} / \mathrm{sec}$, is not a classical shortperiod mode. This mode's eigenvector or mode is depicted in Fig. 7. Note that unlike a conventional short-period mode shape for a rigid vehicle, the second largest contributor to this modal response is centerbody pitch rate due to the first elastic degree of freedom, indicated by $\dot{\theta}_{E 1}$. So as in a true short-period mode, there is virtually no surge velocity $u_{\text {rig }}$ present in this mode, the mode is dominated by the rigid-body (i.e., mean axis) pitch-rate response of the vehicle $q_{\text {rig }}$, and the phase relationships between rigid-body pitch rate, pitch attitude, and angle of attack are as in a conventional short-period mode. But this elastic-short-period mode is a highly coupled rigid-body and elastic mode.

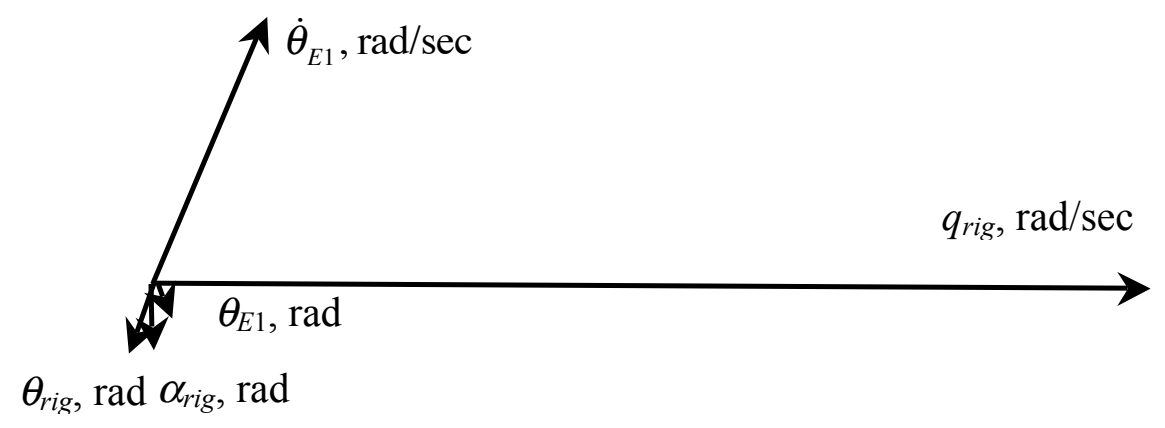

\section{Figure 7, "Elastic-Short-Period” Mode Eigenvector (Mode Shape)}

The next-highest-frequency mode reflected in the transfer functions is the lightly damped first aeroelastic mode (AE Mode 1), with an undamped natural frequency of approximately 31 $\mathrm{rad} / \mathrm{sec}$. This mode's eigenvector (or mode shape) is depicted in Fig. 8, also indicating a coupled 
rigid-body/elastic mode, but dominated by the centerbody elastic pitch-rate deformation associated with the first elastic (bending-torsion) degree of freedom, $\dot{\theta}_{E 1}$. The next largest contributor to this modal response is the rigid-body pitch rate (mean axis) $q_{\text {rig }}$, and there is again virtually no surge velocity $u_{\text {rig }}$ present in this modal response. So as with the elastic-short-period mode in Fig. 7, this first aeroelastic mode also exhibits extensive rigid-elastic coupling.

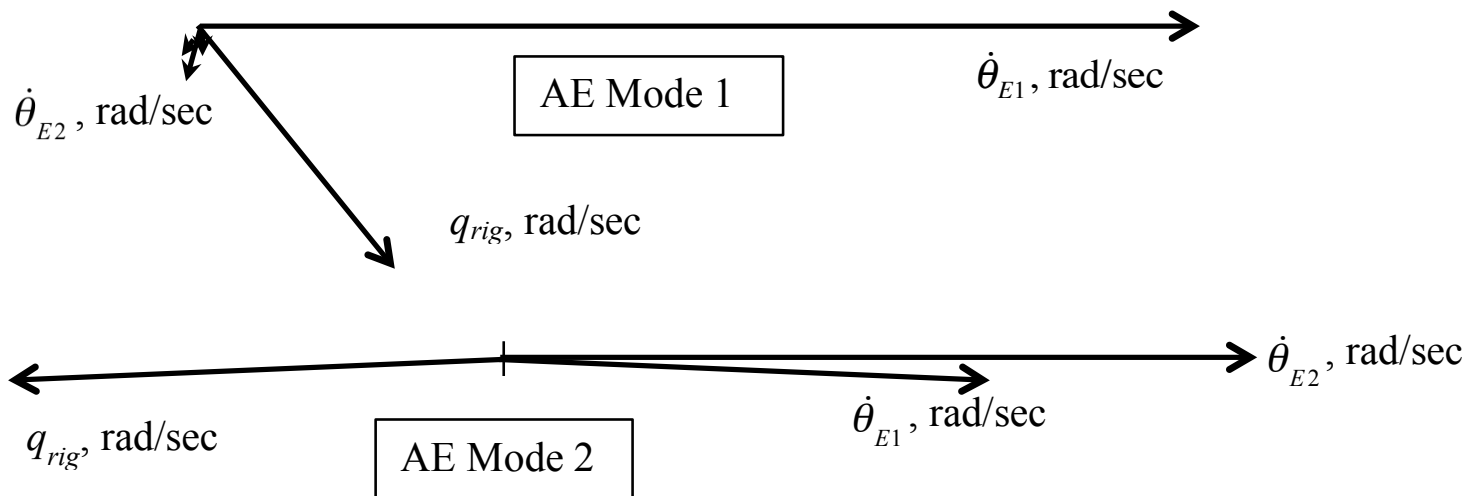

\section{Figure 8, Coupled First and Second Aeroelastic Mode Eigenvectors (Mode Shapes)}

The next-highest-frequency mode is the second aeroelastic mode (AE Mode 2), with an undamped natural frequency of approximately $104 \mathrm{rad} / \mathrm{sec}$. This mode's eigenvector (or mode shape) is also depicted in Fig. 8. Recall the genesis of this mode was a pure torsional vibration mode. This mode is now also a coupled rigid-body/elastic mode, but is dominated by the centerbody pitch-rate associated with the second elastic (torsion) degree of freedom $\dot{\theta}_{E 2}$. The next largest contributors to this modal response are the rigid-body pitch rate $q_{\text {rig }}$ and the elastic pitch rate associated with the first elastic degree of freedom $\dot{\theta}_{E 1}$. It is interesting to note that in this modal response the centerbody-pitch-rate associated with the first two elastic degrees of freedom $\dot{\theta}_{E 1}$ and $\dot{\theta}_{E 2}$ are almost perfectly in phase, while the rigid-body pitch rate $q_{r i g}$ is almost 180 
degrees out of phase with these two elastic responses. This second aeroelastic mode also exhibits significant rigid-elastic coupling.

The last mode is the third aeroelastic mode (mode shape not plotted) with an undamped frequency of $146 \mathrm{rad} / \mathrm{sec}$, and it is almost entirely elastic centerbody-pitch-rate deformation associated with the third elastic degree of freedom, or $\dot{\theta}_{E 3}$. So this is almost a pure elastic mode.

Now consider Fig. 9, which shows the centerbody-pitch-rate step responses from a negative-one-degree elevator deflection $\delta_{3}$, from the three different dynamic models. The response shown in blue is for the rigid vehicle, obtained from truncating all three elastic degrees of freedom, the response in red is the response from a reduced-order model obtained by residualizing all three elastic degrees of freedom, and the response shown in orange is from the full-order NDOF model corresponding to Eqns. 3. These three responses differ significantly, indicating the degree of flexibility of this vehicle. The pitch-rate responses from the models that include the effects of the elastic deformation are quicker, with peak times about 40 percent less than that of the rigid model.

As the final topic in this section we will again consider the vehicle's modal characteristics (eigenvalues and eigenvectors), except at a flight condition above the flutter speed. The longitudinal eigenvectors and eigenvalues for the $50 \mathrm{kt}$ flight condition are shown in Fig 10. These modal characteristics clearly indicate that the vehicle is very flexible, and that the rigidbody and elastic degrees of freedom are highly coupled. And in addition to the system now being unstable, these eigenvectors differ significantly from those shown in Figs. 7 and 8. Most notably, although it appears that an "elastic-short-period mode" is still present, in this mode the response 


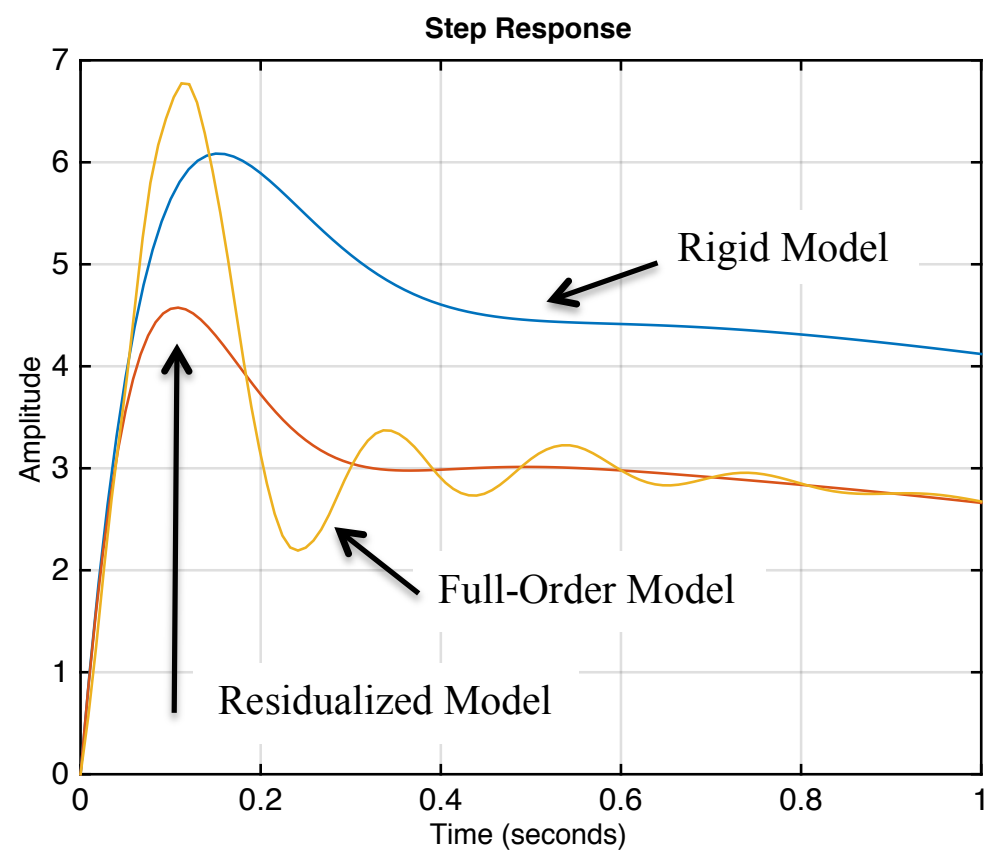

Figure 9, Pitch-Rate Step Response From Del3 (deg/sec/deg)

associated with the rate of change of the first elastic degree of freedom $\dot{\theta}_{E 1}$ now lags the rigidbody pitch rate instead of leading it, as in Fig. 7; in the first-aeroelastic (unstable BFF) mode, the lag of the rigid-body pitch rate behind the rate of change of the first elastic degree of freedom $\dot{\theta}_{E 1}$ is increased; and in the second-aeroelastic (torsion) mode, the dominant response is now that associated with the rate of change of the first elastic degree of freedom $\dot{\theta}_{E 1}$, plus the rigid-body pitch rate is now in phase with the two elastic response rates. So with increased flight velocity, there is more coupling between the rigid-body and elastic degrees of freedom in the modes, and the phase relationships in the mode shapes between the various degrees of freedom are modified. 


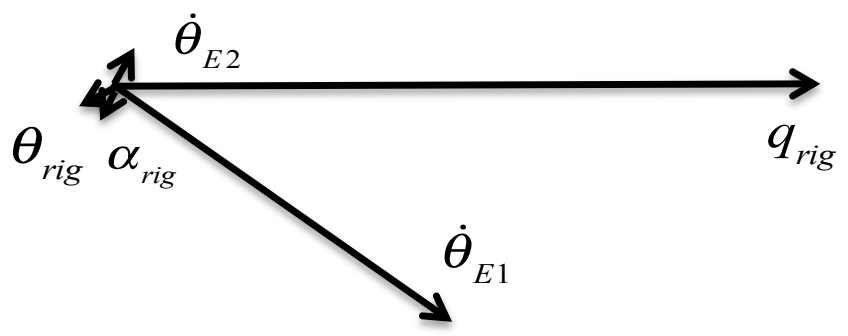

$$
\begin{gathered}
\text { Elastic Short Period } \\
\lambda=-28.5 \pm 24.1 j
\end{gathered}
$$

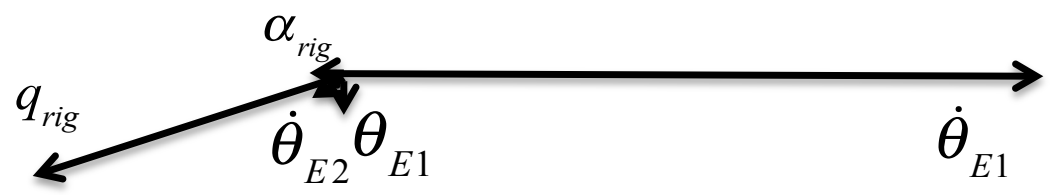

$\underline{1^{\text {st }} \text { Aeroelastic - BFF }}$

$\lambda=1.9 \pm 27.8 j$

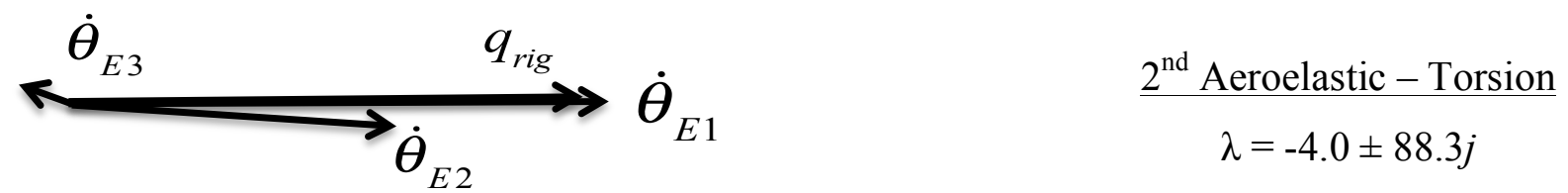

Figure 10, Modal Characteristics at the 50k Flight Condition - Unstable

\section{Actuator-Sensor Pair Selections}

The overall approach to be taken in the control synthesis is motivated by the concept known as Identically Located Force and Acceleration (ILAF), successfully utilized in the development of the active-structural-mode control system on the B-1 aircraft (Ref. 8), and others. ILAF essentially states that a force applied to a point on the structure proportional to the velocity of the structure measured at the point of application of the force will increase the damping of all structural modes. This concept is powerful since it does not rely on precise knowledge of the structural mode shapes, which are difficult to predict accurately and change with changes in mass distribution and stiffness properties of the structure.

The basic idea behind ILAF stems from Eqn. 2 above, or

$$
\ddot{\eta}_{i}(t)+2 \zeta_{i} \omega_{i} \dot{\eta}(t)+\omega_{i}^{2} \eta_{i}=Q_{i} / \mathbb{M}_{i}, \quad i=1, \ldots, n
$$

where all the terms in this equation are defined below Eqn. 2. Recall that this is the equation of motion governing the $i^{\prime}$ th elastic degree of freedom associated with the $i$ 'th free-vibration mode of the vehicle's structure. The generalized force $Q_{i}$ exciting this degree of freedom is due to the 
forces acting on the vehicle. Now consider some point force $\mathbf{F}$ acting on the structure at location $x_{F}, y_{F}, z_{F}$ on the undeformed vehicle. The generalized force in this case can be expressed in terms of the dot product between the applied force $\mathbf{F}$ and the vibration mode shape (expressed as a vector quantity), or

$$
Q_{i}=\mathbf{v}_{i}\left(x_{F}, y_{F}, z_{F}\right) \bullet \mathbf{F}
$$

where the mode shape $\mathbf{v}_{i}$ was introduced in Eqn. 1.

Now assume that the force $\mathbf{F}$ is strictly proportional to the local velocity of the structure measured at the point $x_{F}, y_{F}, z_{F}$. Or in terms of the structural deformation $\mathbf{d}_{E}$ in Eqn. 1, let

$$
\mathbf{F}=\left.K \frac{d \mathbf{d}_{E}}{d t}\right|_{b o d y}\left(x_{F}, y_{F}, z_{F}\right)
$$

where $K$ is some negative constant of proportionality, and the time rate of change of $\mathbf{d}_{E}$ is taken with respect to the vehicle's body-fixed coordinate frame. So from Eqn. 1 again we see that this force $\mathbf{F}$ may be written as

$$
\mathbf{F}=\left.K \frac{d \mathbf{d}_{E}}{d t}\right|_{b o d y}\left(x_{F}, y_{F}, z_{F}\right)=K \sum_{i=1}^{n} \mathbf{v}_{i}\left(x_{F}, y_{F}, z_{F}\right) \dot{\eta}_{i}(t)
$$

And therefore, the generalized force $Q_{i}$ becomes simply

$$
Q_{i}=K \mathbf{v}_{i}\left(x_{F}, y_{F}, z_{F}\right) \bullet \sum_{j=1}^{n} \mathbf{v}_{j}\left(x_{F}, y_{F}, z_{F}\right) \dot{\eta}_{j}(t)=K^{\prime} \dot{\eta}_{i}(t)+\sum_{\substack{j=1 \\ j \neq i}}^{n} K_{j} \dot{\eta}_{j}(t)
$$

with $K^{\prime}$ strictly non positive and $K_{j}=0$ for all $j \neq i$ if the mode shapes are orthogonal.

Now if one inserts this expression into Eqn. 5 above for the $i$ 'th mode, we see that the damping of this elastic mode will always be increased unless $\mathbf{F}$ happens to be applied at a node where $\mathbf{v}_{i}\left(x_{F}, y_{F}, z_{F}\right)=\mathbf{0}$. And in like manner the damping of all $i$ elastic modes will be increased. Of course the practical issues include the measurement of the local velocity of the structure, the application of a point force, and the finite bandwidth of the force actuator. 
From the discussion in Sections 3 and 4 above, one can observe that the crux of the fluttersuppression problem for this vehicle is the interactions between the vehicle's rigid-body pitching and the first symmetric bending/torsion mode, as well as the second flutter condition involving the second symmetric torsion mode. And a key will be the judicious selection of sensor-actuator combinations to be used. This selection will be guided by the ILAF concept and accomplished via logic and basic feedback techniques, rather than any mathematic algorithms. The thought process is as follows:

1. We seek to increased damping of all aeroelastic modes and to do so robustly against changes in mass distribution and/or stiffness properties - so we will apply ILAF.

2. The increased damping realized through the application of ILAF is theoretically achieved regardless of the point of velocity measurement and force application on the structure. But the co-located force and measurement will be most effective at damping modes with mode shapes such that the modes are both highly observable in the velocity measurement and highly controllable from a force applied at that location. That is, the location chosen on the structure for measurement and force application should not lie near node lines for particularly troubling (e.g., unstable) modes.

3. The BFF condition involves the interactions between the vehicle-pitching mode (elasticshort-period) and the first aeroelastic mode that involves both bending and significant pitching/wing twist (see Fig. 3). And of course, the elastic-short-period mode involves pitching of the center body and wing. Finally, as suggested by the vehicle mode shape in Fig. 8, pitch coupling is key to BFF. 
4. So as a corollary to ILAF, applying a pure pitching moment to a point on the structure proportional to the pitch rate measured at the same location on the structure should be effective at suppressing body-freedom flutter.

5. But note that true ILAF is not possible for this vehicle as configured. ILAF requires applying a force (or a torque) at a point on the structure, but the control surfaces generate distributed loads over the wing or center body. Plus the trailing-edge control surfaces generate both wing twist (torque) as well as increased lift, not the pure pitching moment called for by ILAF. The center-body flaps, however, apply a distributed load over a region of the structure over which the mode shapes (Figs. 3-5) are constant (i.e., the center body), which is desirable. So this body-flap/pitch-rate force-measurement pair are not perfect, but perhaps sufficiently close to ILAF to be successful.

6. This hypothesis is supported by the results shown in Fig. 11. (The flight condition is $50 \mathrm{kt}$ at $3000 \mathrm{ft}$,) Note that the locations of the zeros in the aeroelastic dipoles are such that damping of all modes is increased. Plus, this loop closure is especially effective at stabilizing the BFF.

7. The mode least affected by this loop closure is the second aeroelastic mode, with genesis consisting of the first-torsion vibration mode. Note from its mode shape in Fig. 4 that this mode exhibits little center-body pitch (twist) and essentially no bending. So the mode is not very observable or controllable with regard to this body-flap/pitch-rate loop. Recall that this mode eventually becomes unstable at higher dynamic pressure, so it must be actively controlled through a second loop closure. But since this mode should not be greatly affected by the body-flap/pitch-rate loop, the control-law synthesis should be simpler. 


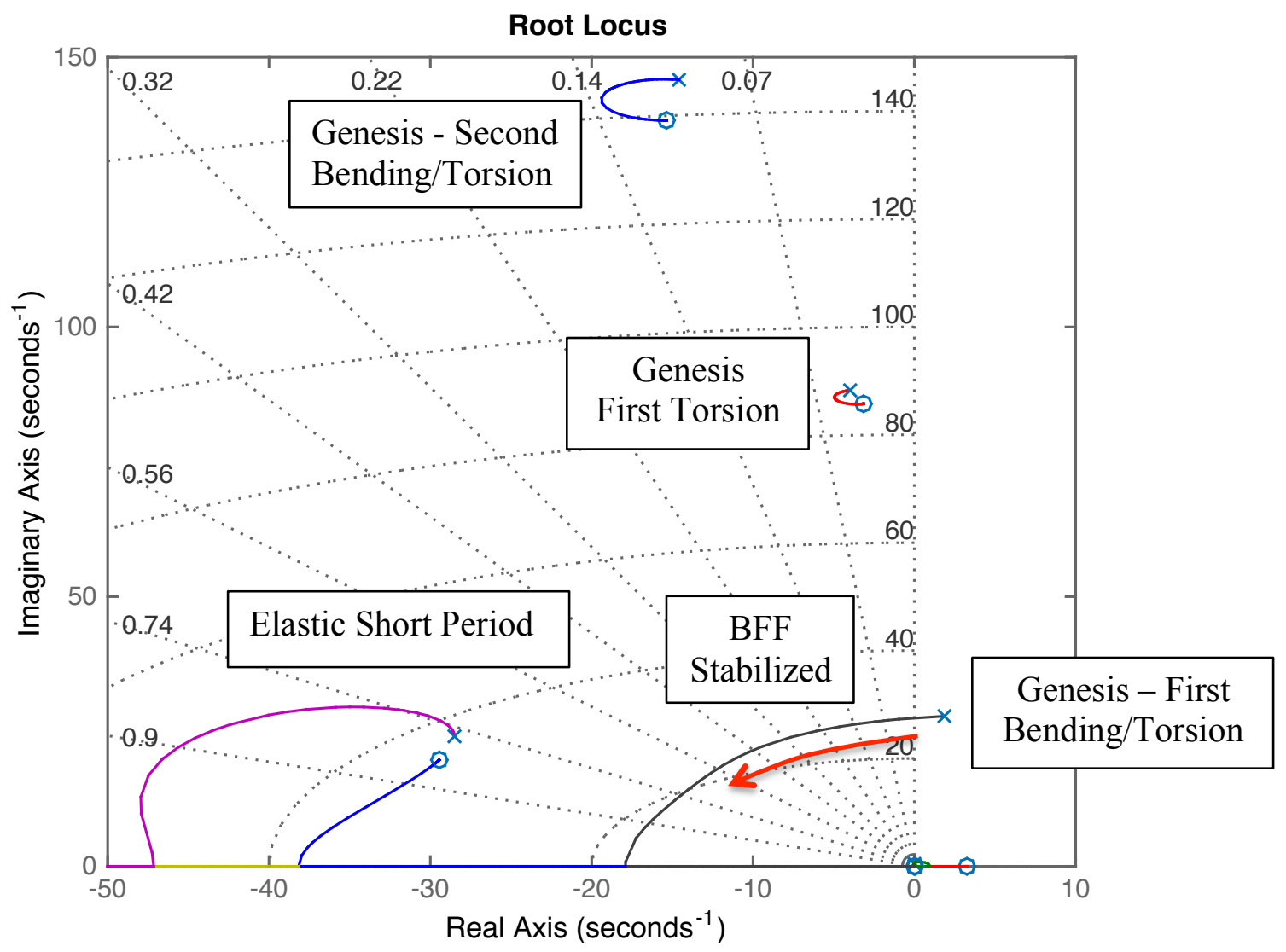

Figure 11, Root Locus - Center-Body Pitch Rate From Body-Flap Deflection $\left(q_{C B} /-\delta_{B F}\right)$

8. With regard to this second loop closure, note from the mode shape (Fig. 4) that this firsttorsion mode will be very observable in wing-tip twist measurements and very controllable from the outboard control surfaces, which impart a twisting torque on the wing. So closing a loop on wing twist to the outboard surfaces should be effective at augmenting the second aeroelastic mode.

9. This assertion is verified by the root locus shown in Fig. 12 (same flight condition: $50 \mathrm{kt}$, $3000 \mathrm{ft}$ altitude), which corresponds to symmetric wing-twist acceleration $\ddot{\theta}_{t w i s t}$ fed back to the outboard surfaces. Note that this second loop closure is not a true ILAF loop, as 
discussed earlier under Item 5 above, plus twist acceleration is being fed back, rather than twist velocity. So the damping of all modes may not be increased, but the torsion-mode damping will be significantly increased, as desired. Note that this loop closure also tends to stabilize the BFF, but that is not its primary purpose. With a low loop gain the damping of the first torsion mode should be sufficiently increased, without excessively destabilizing the elastic-short-period mode. Differencing the two wing-tip accelerometers, one at the wing's leading edge and one at the hinge line of the outboard control surface may be used to sense wing-tip twist acceleration.

Consequently, a properly tuned control scheme that both feeds back center-body pitch rate to the body flaps as well as differenced wing-tip accelerometers to the outboard control surfaces should be effective at both suppressing BFF as well as increasing the damping of the first-torsion mode as needed. Plus, there is an additional benefit of feeding pitch rate to a pitch control surface. It is known (c.f., Ref. 3) that such a feedback loop is both effective at increasing the damping of a conventional short-period mode, plus it preserves conventional aircraft-like dynamics. This is in contrast to some attitude controllers that contaminate conventional aircraft-like modal characteristics. Hence, the pitch-rate-feedback loop is also effective as a rigid-body stabilityaugmentation system (SAS). Therefore, the control architecture depicted in Fig. 13 is proposed, and will now be consider in more detail. 


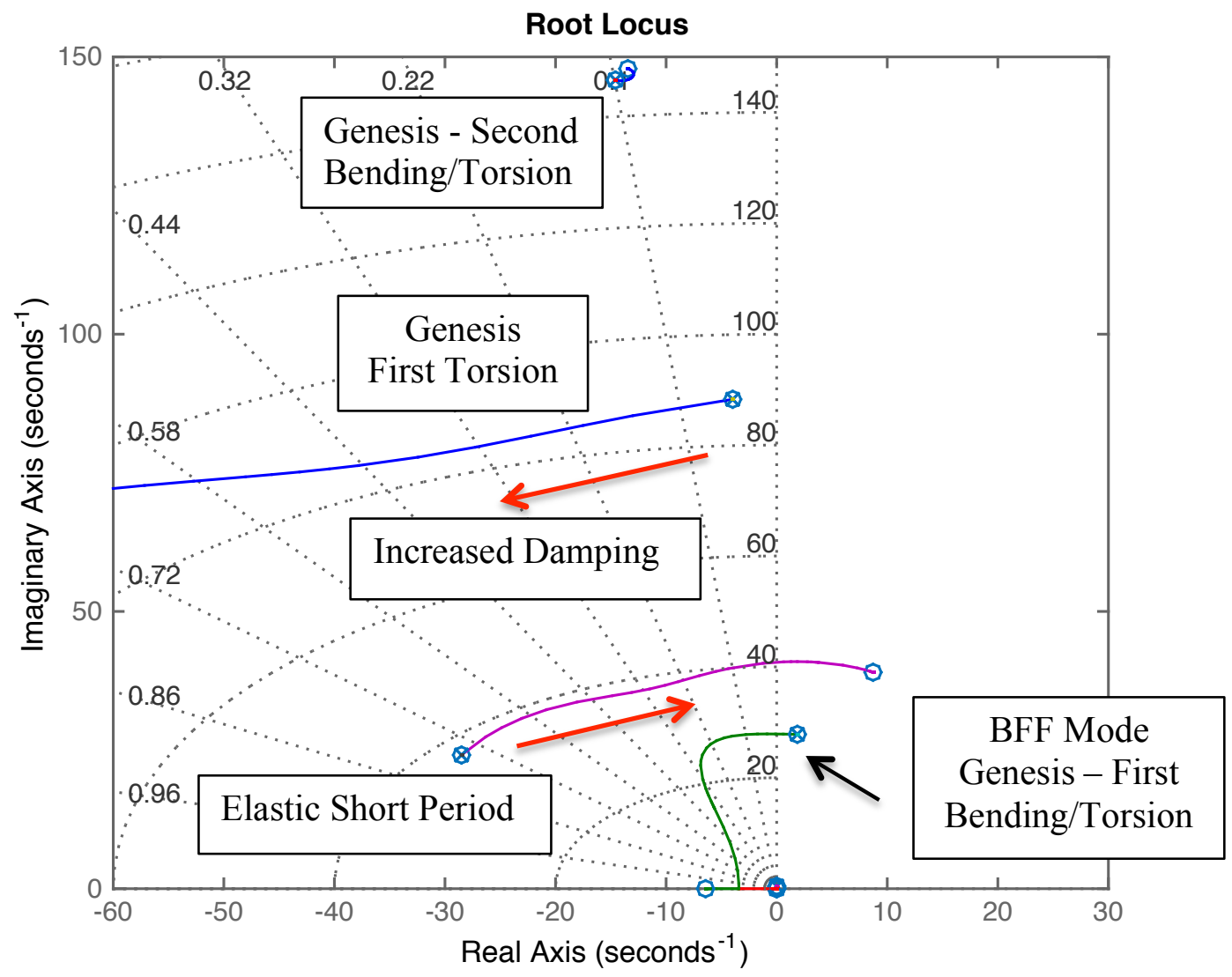

Figure 12, Root Locus - Wing-Tip Twist From Del-4 Deflection $\left(\dot{q}_{T i p} /-\delta_{4}\right)$

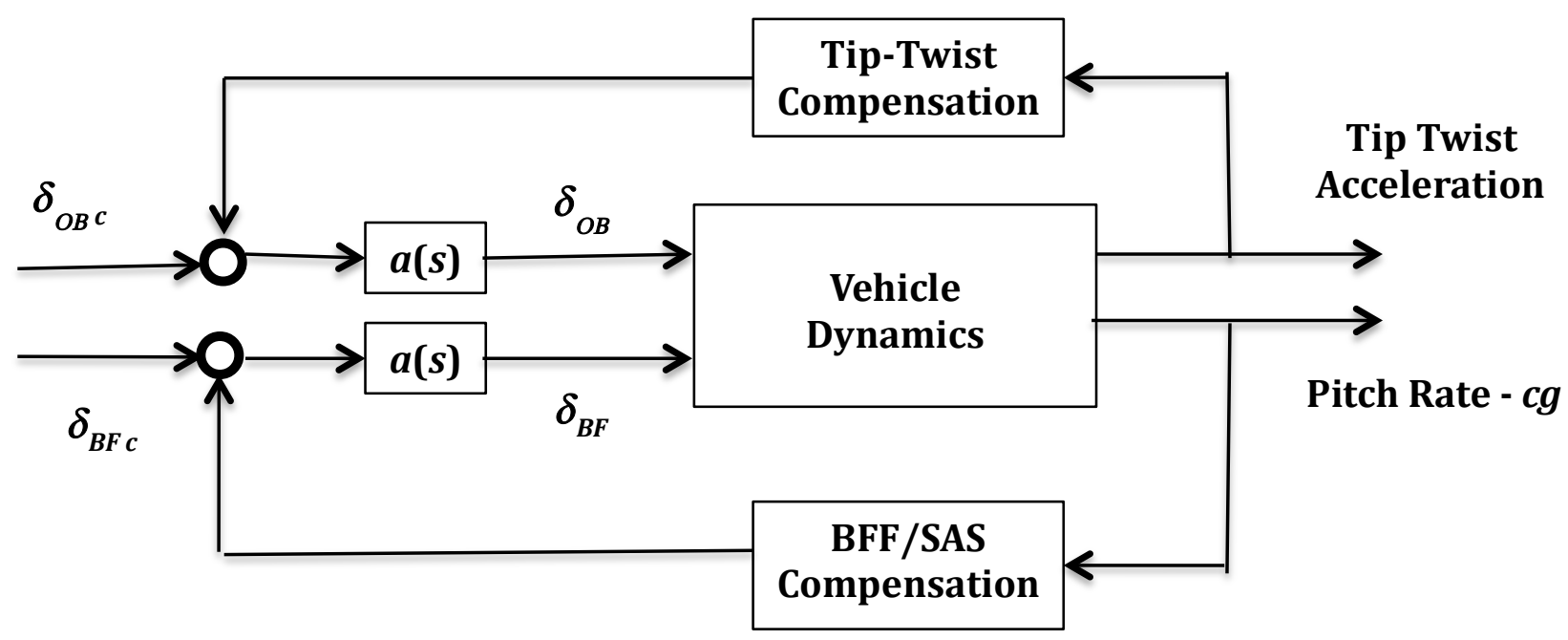

Figure 13, Suggested Feedback Architecture 


\section{Control-Law Synthesis and Analysis}

This analysis addresses both the $50 \mathrm{kt}$ and $60 \mathrm{kt}$ flight conditions, the first one being below the torsion flutter speed and only the BFF condition is unstable. Only the $50 \mathrm{kt}$ case will be considered in detail here, with the results for the $60 \mathrm{kt}$ flight condition summarized later. Conventional frequency-domain techniques and successive loop closure will be employed. Recalling that closure of the pitch-rate/body-flap loop minimally affects the torsion-mode roots, plus observing that the wing-twist loop will have the higher crossover frequency of the two loops, suggest that addressing the augmentation of the torsion mode first is advisable.

\subsection{Bare-Airframe Only - 50 Knots}

Ignoring the actuators for now, we take the transfer functions $a(s)$ in Fig. 12 to be unity. Using MATLAB's control-design tool, the particulars of the wing-twist/outboard-flap loop closure (with the body-flap loop open) are revealed in Fig. 14. Three plots are included in the figure, a root locus (identical to that in Fig 12), and the open- and closed-loop frequency responses for this feedback loop. As indicated in the root locus, a modest gain $\left(-7.5 \times 10^{-4}\right.$ $\mathrm{deg} / \mathrm{deg} / \mathrm{sec}^{2}$ ) relocates the augmented poles to the locations indicated, and the damping of the torsion mode is increased from less than 0.05 to 0.20 . The open-loop Bode plot on the right confirms that this loop closure is effective at robustly augmenting the torsion mode, with an infinite gain margin and a phase margin greater than 90 deg at a gain-crossover frequency of around $100 \mathrm{rad} / \mathrm{sec}$. But the loop still retains the neutrally stable BFF mode and the unstable Phugoid mode, both at lower frequencies. We will deal with the BFF mode next.

The second loop closure is the BFF loop, or center-body pitch rate fed back to the body flaps with the tip-twist loop closed. Again the particulars of this loop closure are revealed in Fig. 15, which includes the loop's root locus and open- and closed-loop frequency responses. This root 
locus now differs from that in Fig. 11, due to the tip-twist loop having been closed, and in particular the torsion-mode poles are now farther to the left, as desired.
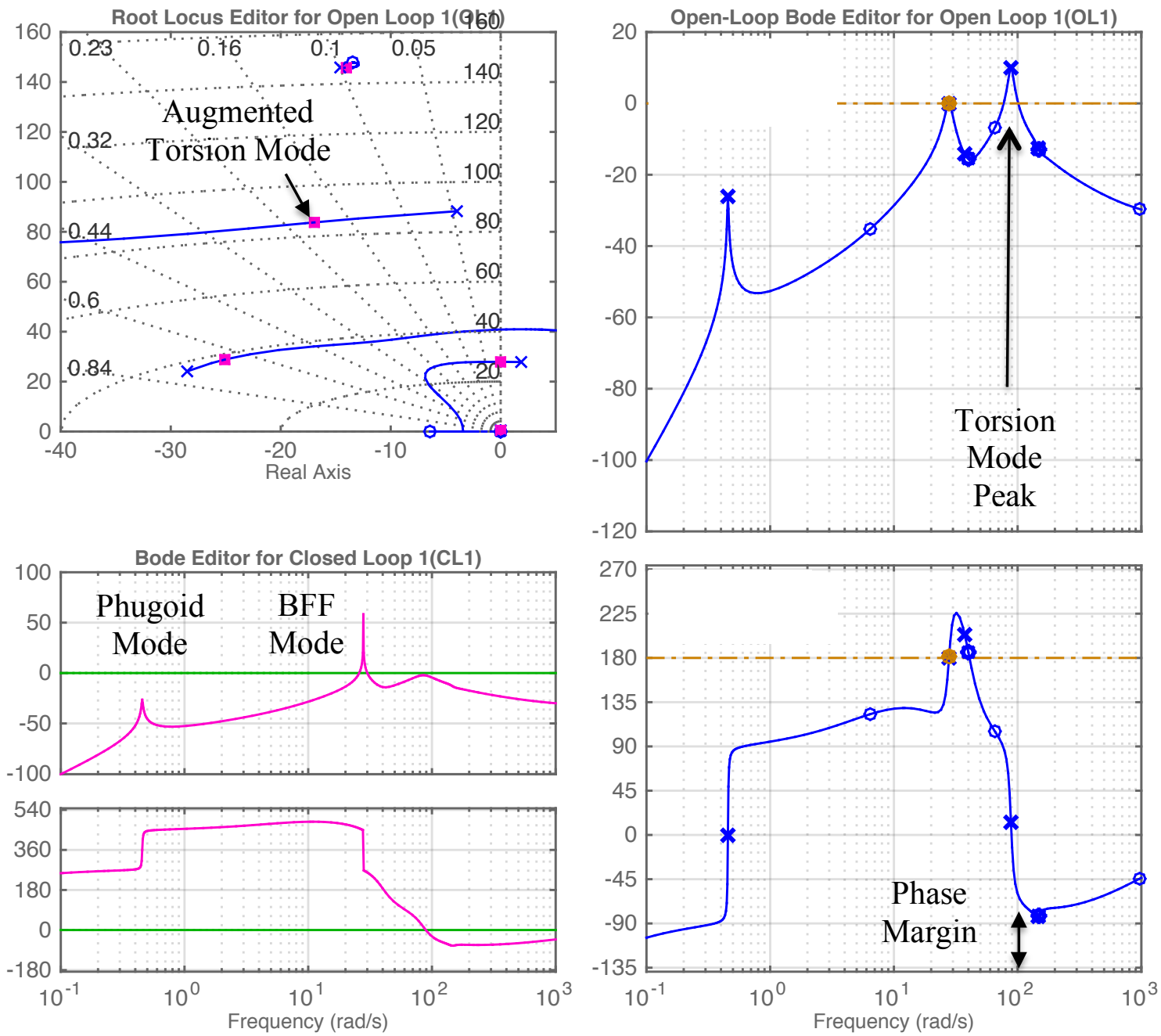

Figure 14, Graphical Analysis of the Tip-Twist Loop Closure (nz-twist/ $\left.\delta_{O B}\right)$

A feedback gain of $-0.28 \mathrm{deg} / \mathrm{deg} / \mathrm{sec}$ moves the augmented poles to the locations indicated. The damping of all modes has been increased, and in particular the BFF mode has been stabilized. The open-loop Bode plot confirms that this loop closure is effective at robustly stabilizing the BFF mode, with a theoretically infinite gain margin and a high-frequency phase margin of greater than $90 \mathrm{deg}$ at a gain-crossover frequency of around $60 \mathrm{rad} / \mathrm{sec}$. Only the 
Phugoid mode remains unstable, but it would of course be stabilized through the pilot's (or autopilot's) control of pitch attitude. The closed-loop eigenvalue locations are given Table 7.
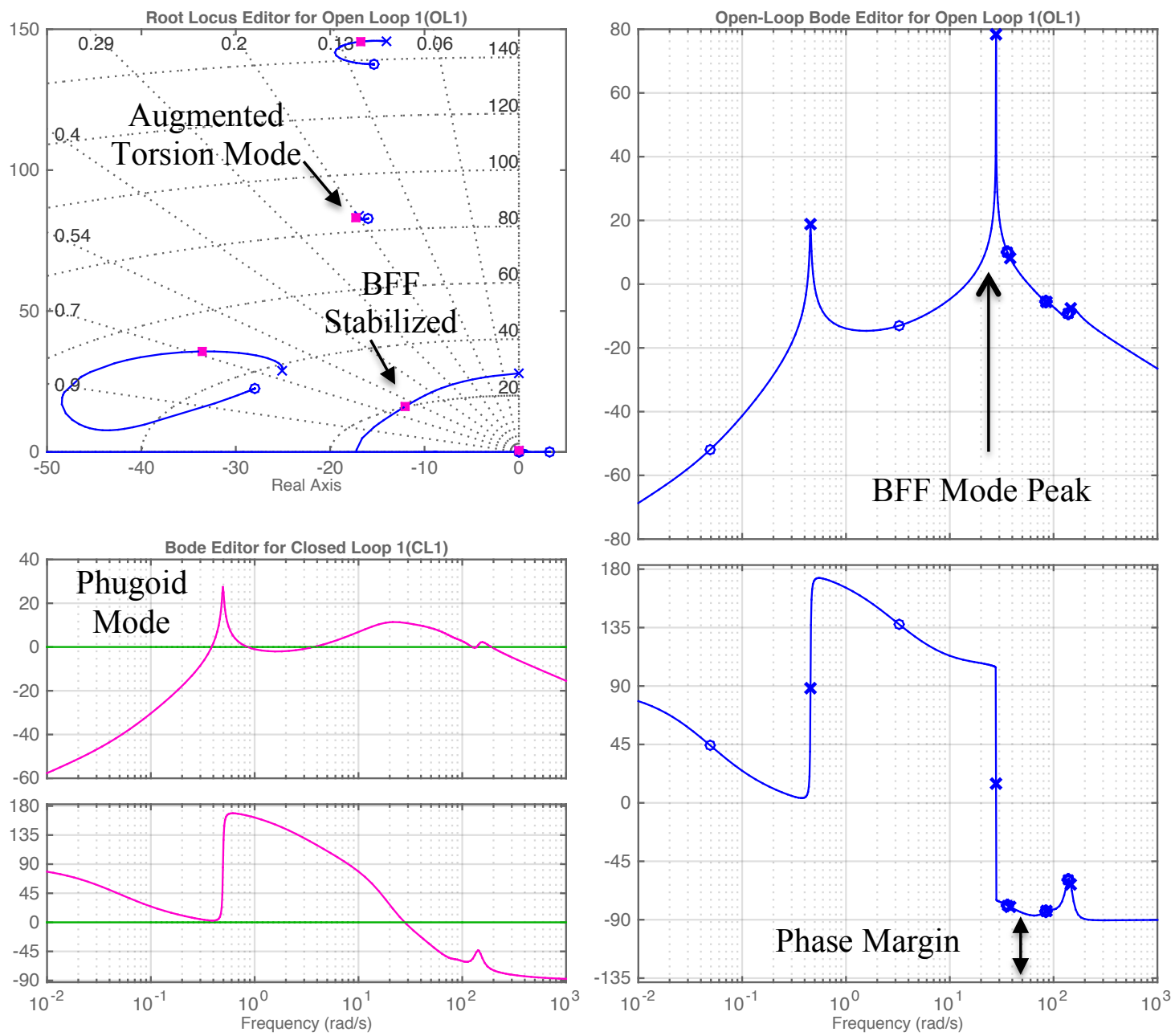

Figure 15, Graphical Analysis of the Pitch-Rate Loop Closure $\left(q_{c g} / \delta_{B F}\right)$

Table 7, Closed-Loop Eigenvalues - Bare Airframe Only

Eigenvalue, r/s $\varsigma \quad \omega, \mathbf{r} / \mathbf{s}$ Root-Locus Branch

\begin{tabular}{|llll|}
\hline$-16.8+/-146 j$ & 0.114 & 147.0 & $3^{\text {rd }}$ Aeroelastic \\
$-17.3+/-83.1 j$ & 0.203 & 84.9 & $2^{\text {nd }}$ Aeroelastic \\
$-33.6+/-35.7 j$ & 0.685 & 49.0 & Elastic Short Period \\
$-12.1+/-16.1 j$ & 0.599 & 20.1 & BFF \\
$0.01+/-0.50 j$ & -0.014 & 0.495 & Phugoid \\
\hline
\end{tabular}


It is interesting to now consider the modal characteristics of the augmented vehicle, as depicted in Fig. 16. The eigenvalues listed in Table 7 are included, to help identify the modes. Note that the modes are ordered in this figure to be consistent with the ordering in Fig. 10, and the modal characteristics here differ from those of the unaugmented vehicle given in Fig. 10. Most notably, in addition to the system being stabilized, the character of the modes has changed considerably. The rigid-body pitch rate is no longer the dominant response in the former elasticshort-period mode, and there is less rigid-body-attitude and angle-of-attack responses in this mode as well. So this mode has less overall rigid-body response in it, when compared to the corresponding mode in Fig. 10, hence this mode no longer dominates the rigid-body response.
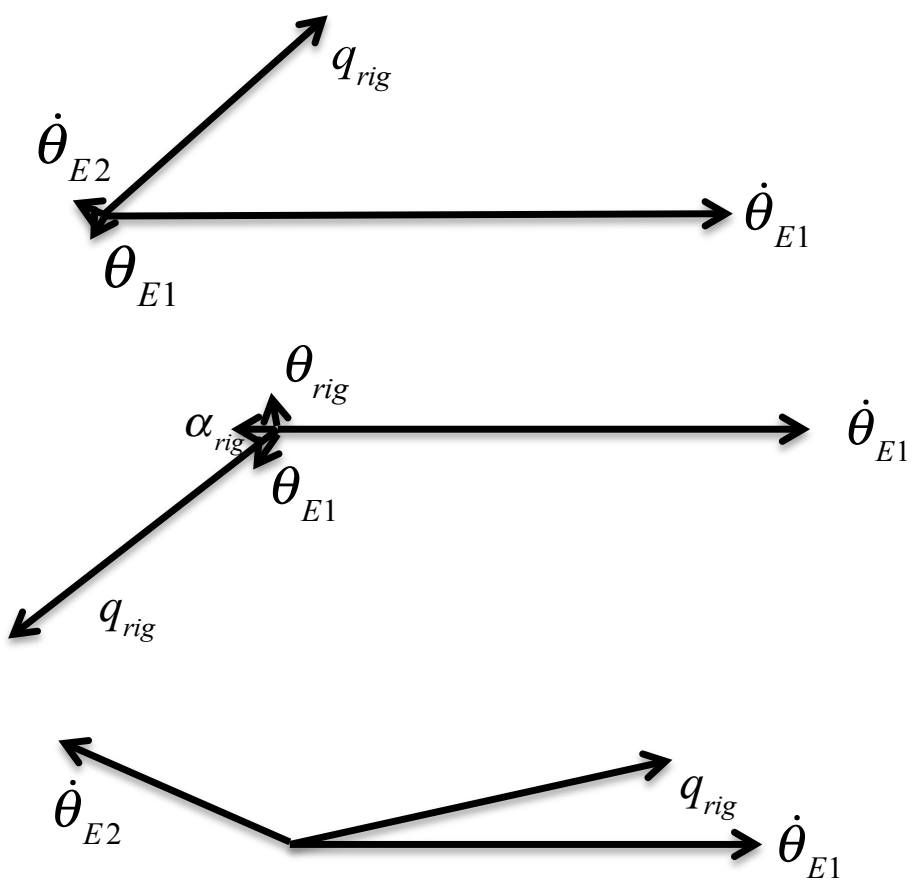

Former Elastic Short Period

$\lambda=-33.6 \pm 35.7$

$\underline{\text { Stabilized BFF }}$

New Elastic Short Period

$$
\lambda=-12.1 \pm 16.1 j
$$

Figure 16, Modal Characteristics of the Augmented Bare Airframe - 50 kt

In contrast, the participation of the rigid-body degrees of freedom $\left(q_{r i g}, \theta_{r i g}\right.$, and $\left.\alpha_{r i g}\right)$ have all increased in the now-stabilized BFF mode. Furthermore, if one considers just the portion of the phasor diagram for this mode that is associated with these three rigid-body responses (i.e., 
ignoring the $\theta_{E 1}$ and $\dot{\theta}_{E 1}$ components), it is very similar to the phasor diagram for a conventional short-period mode for a rigid aircraft. Hence this mode now dominates the vehicle's rigid-body response and has become the new elastic-short-period mode. But the dominant response in this mode is that associated with the first elastic degree of freedom, far different from any conventional short-period mode.

\subsection{Effect of the Actuators - 50 Knots}

All the results above only considered the dynamics of the bare airframe. They do not include the effects of the finite bandwidth of the surface actuators. And we shall see that the actuators have a significant effect on the results. Based on information provided by Lockheed Martin, the dynamics of the actuators are approximately given by

$$
\frac{\delta(s)}{\delta_{c}(s)}=\frac{15,790}{s^{2}+163.4 s+15,790}
$$

With reference to the block diagram in Fig. 13, $a(s)$ now becomes the above transfer function, and we will re-examine our two control loops.

The particulars of the wing-twist/outboard-flap loop closure (with the body-flap loop open) are revealed in Fig. 17. The root locus in this figure is considerably different from that shown in Fig. 14, which ignored the actuator dynamics. Now the maximum achievable increase in torsion-mode damping is limited, and if the loop gain is too high this mode eventually goes unstable.

A modest gain $\left(-4 \times 10^{-4} \mathrm{deg} / \mathrm{deg} / \mathrm{sec}^{2}\right)$ relocates the augmented poles to the locations indicated, and the damping of the torsion mode is doubled from approximately 0.04 to approximately 0.08 . The open-loop Bode plot on the right confirms that this loop closure augments the torsion mode with gain margin greater than $20 \mathrm{~dB}$ and a phase margin of almost 90 
deg at a gain-crossover frequency of around $90 \mathrm{rad} / \mathrm{sec}$. These margins are also shown in the loop's Nyquist plot given in Fig. 18, and the loop still retains the unstable BFF and Phugoid modes. The key effect of the actuators is the additional phase loss at the higher frequencies. Comparing the phase portion of the open-loop Body plot in Fig. 17 with that in Fig. 14 reveals this loss of phase near the crossover frequency, which severely limits the achievable performance of this loop.
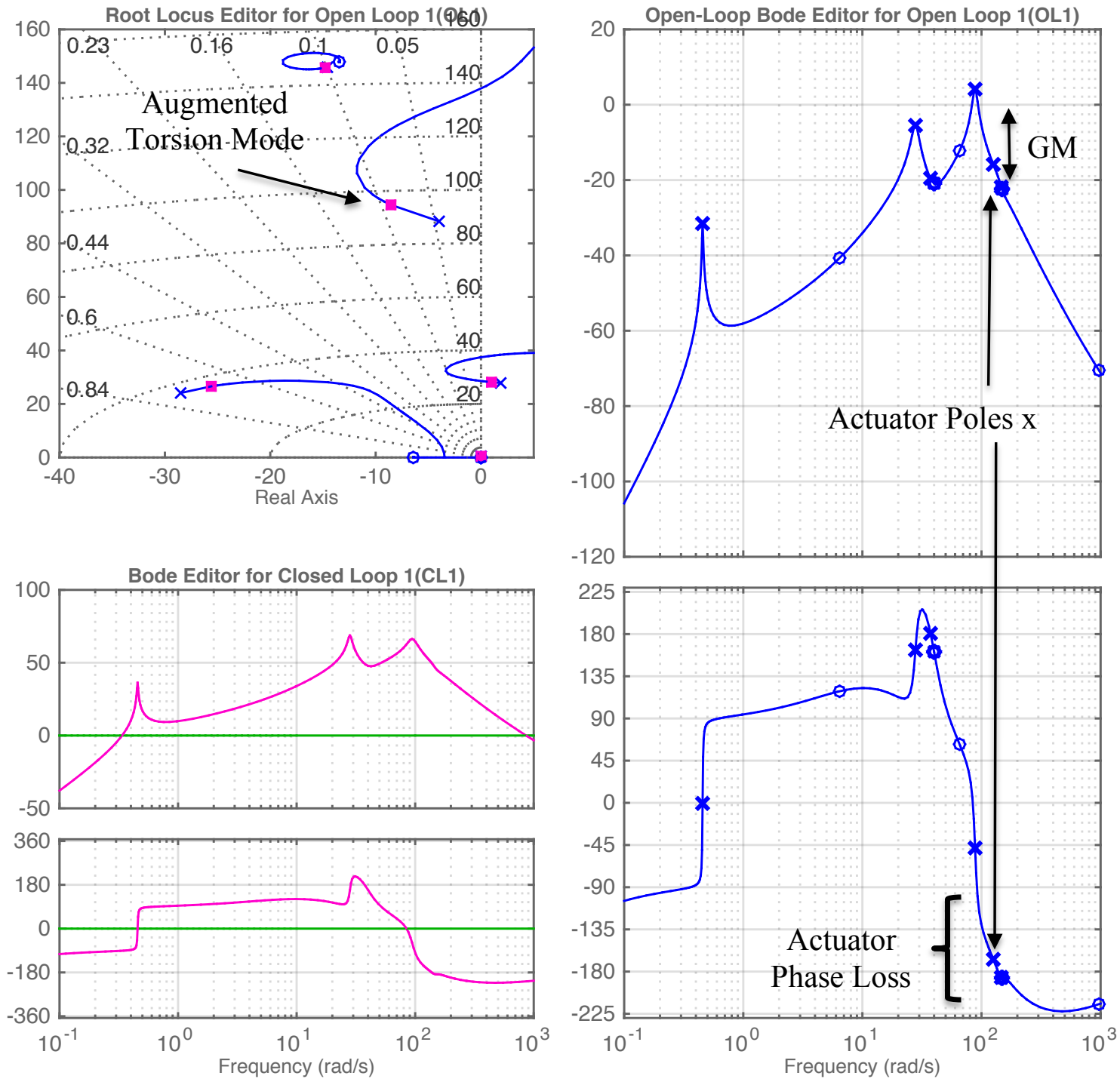

Figure 17, Graphical Analysis of the Tip-Twist Loop Closure (nz-twist/ $\left.\delta_{O B c}\right)-$ w. Actuators 


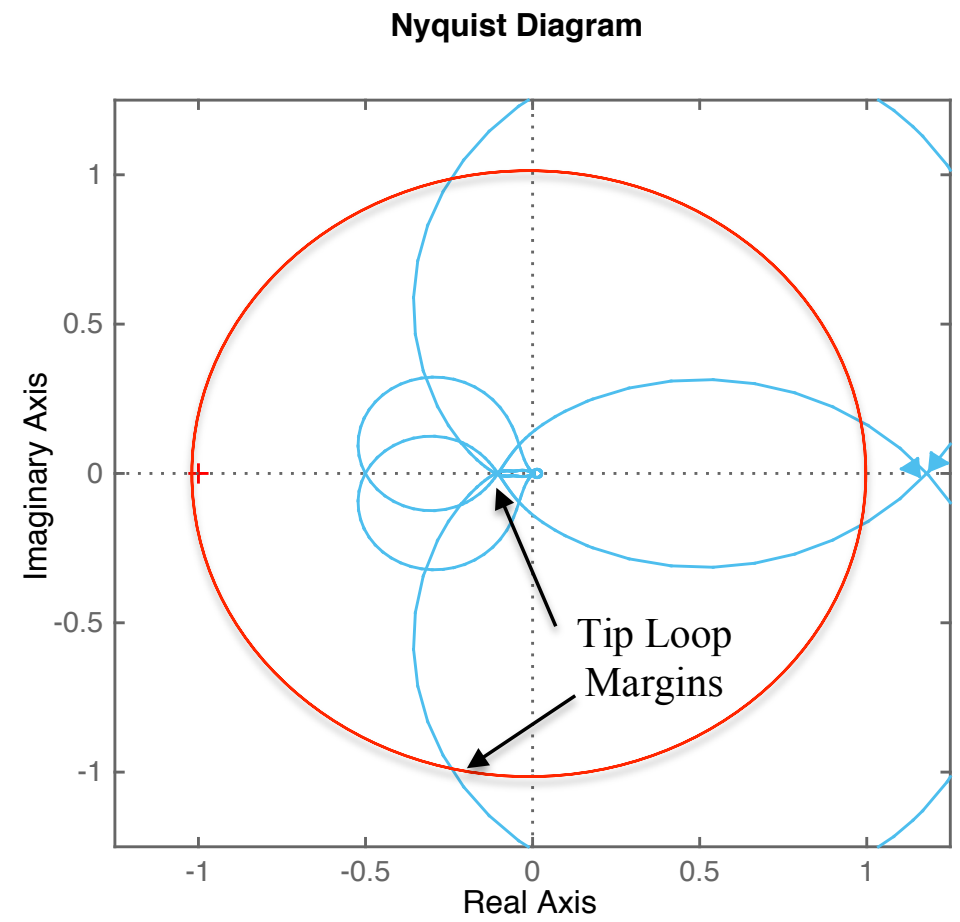

Figure 18, Tip Loop Nyquist - w. Actuators

Looking again at the second loop closure now, or center-body pitch rate fed back to the body flaps with the above tip-twist loop closed, the results are revealed in Fig. 19. The root locus again differs significantly from that in Fig. 15, due to the effects of the actuators. With the addition of the actuators the desirable properties of an ILAF feedback loop are not as clearly evident. The loop closure can still stabilize the BFF instability, but now the third aeroelastic mode can be destabilized with excessive loop gain, and the actuator roots become involved.

Still considering the root locus in Fig. 19, a feedback gain of $-0.20 \mathrm{deg} / \mathrm{deg} / \mathrm{sec}$ moves the augmented poles to the locations indicated in. The BFF mode has been stabilized, the damping of the torsion mode is slightly further increased, and the natural frequency of the mode that began as the elastic short period has been increased to about $50 \mathrm{rad} / \mathrm{sec}$. The open-loop Bode plot in Fig. 19 confirms that this loop closure is effective at stabilizing the BFF mode, with gain margins of -20 and $+14 \mathrm{~dB}$ and phase margins of -60 and $+50 \mathrm{deg}$, and an upper gain-crossover frequency of 
around $45 \mathrm{rad} / \mathrm{sec}$. The positive margins are also shown in the loop's Nyquist plot in Fig. 20. Once again, these margins are limited by the high-frequency phase loss from the actuators. Only the Phugoid mode now remains unstable. The closed-loop eigenvalues are given in Table 8.
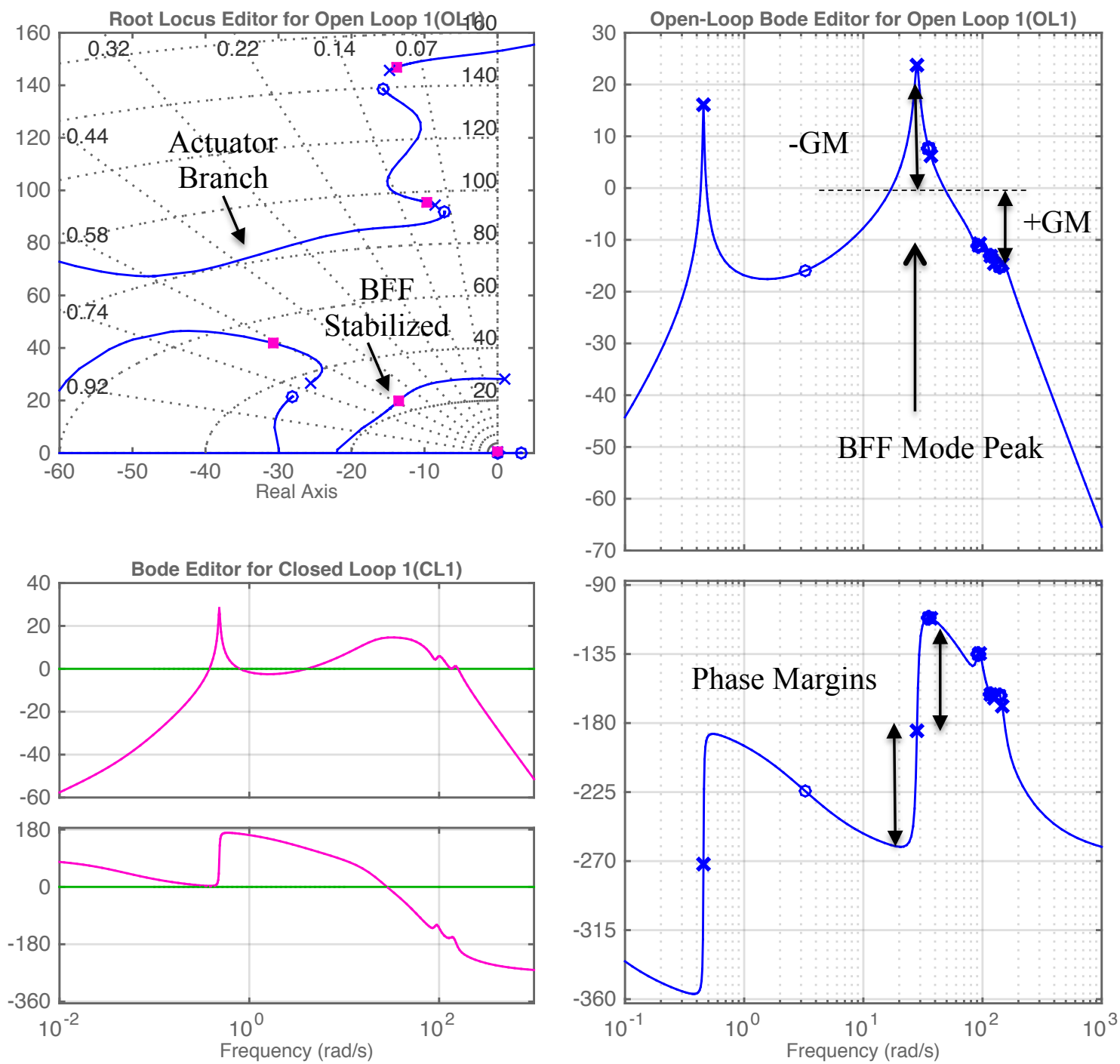

Figure 19, Graphical Analysis of the Pitch-Rate Loop Closure $\left(q_{c g} / \delta_{B F c}\right)-$ w. Actuators 


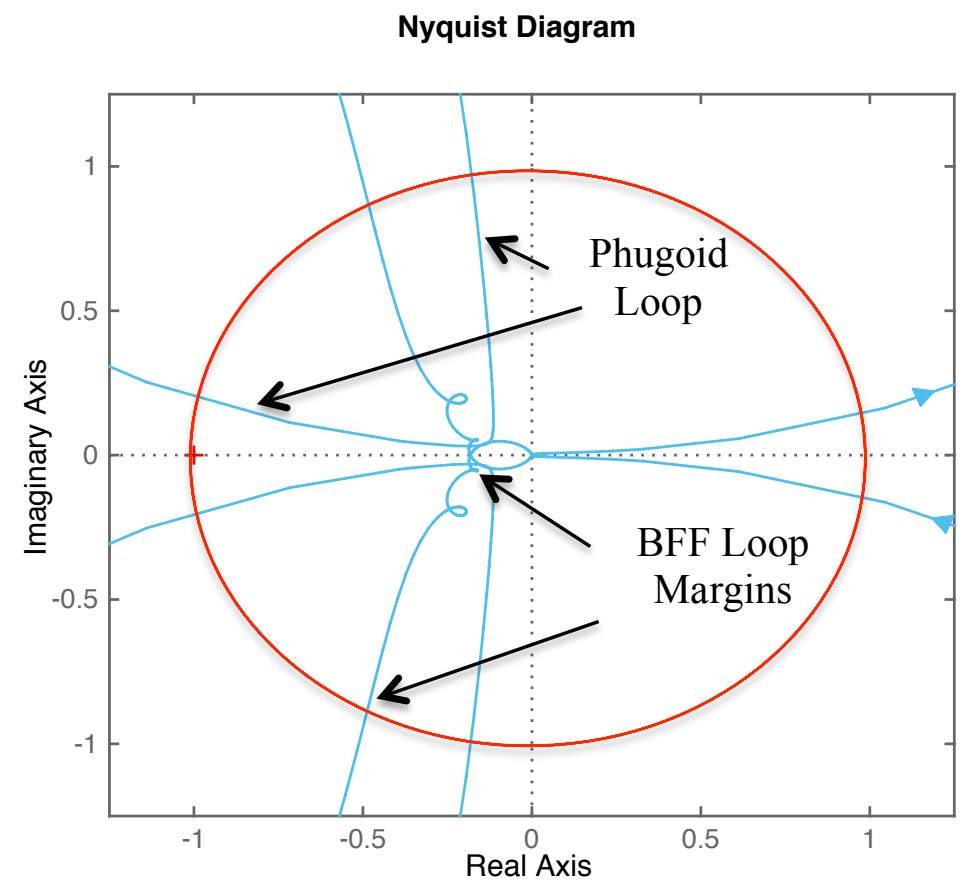

Figure 20, BFF Loop Nyquist - w. Actuators

Table 8, Closed-Loop Eigenvalues - With Actuators

Eigenvalue, r/s $\varsigma \quad \omega, r / s \quad$ Root Locus Branch

$-13.8+/-147 i \quad 0.094 \quad 147 \quad 3^{\text {rd }}$ Aeroelastic

$-78.4+/-86.7 \mathrm{i} \quad 0.671 \quad 117$ Actuator

$-62.4+/-74.7 \quad 0.641 \quad 97.4$ Actuator

$-9.7+/-95.4 \mathrm{i} \quad 0.101 \quad 95.9 \quad 2^{\text {nd }}$ Aeroelastic (Torsion)

-30.7+/- 41.9i 0.59151 .9 Former Elastic Short Period

$-13.5+/-19.9 \quad 0.563 \quad 24.0 \quad$ BFF $\rightarrow$ New Elastic Short Period

$0.006+/-0.48 \mathrm{i} \quad-0.012 \quad 0.48$ Phugoid

We must now check the stability margins in the first loop closure, or tip twist to outboard flap, but with the just designed pitch-rate-to-body-flap loop closed. The gain margin in this case is now $20 \mathrm{~dB}$, while the phase margin is $90 \mathrm{deg}$ measured at a gain-crossover frequency of about 92 $\mathrm{rad} / \mathrm{sec}$. These margins are essentially unchanged from those found in Figs. 17 and 18, which did not include the pitch-rate loop closure. Conversely, consider the margins in the second loop closure, or pitch-rate-to-body-flap, but with the tip-twist loop open. The gain margins are -18 and 
$+15 \mathrm{~dB}$, while the phase margins are -60 and $+50 \mathrm{deg}$ with a higher gain-crossover frequency of about $45 \mathrm{rad} / \mathrm{sec}$. These margins are very close to those found in Figs.19 and 20, which included the tip-twist loop closure. Both these sets of results are reassuring.

The question now turns to the attitude response of the augmented vehicle. Consider the one-degree mid-flap step responses in Figs. 21a and 21b. The responses for center-body pitch rate and plunge acceleration, both measured at the cg location of the undeformed vehicle, are shown along with those for the rigid-body (mean-axis) angle of attack and the body- and outboard-flap control deflections. The attitude responses are clearly dominated by the new elastic-short-period mode with damping equal to 0.56 and a frequency of $24 \mathrm{rad} / \mathrm{sec}$, and there is some modest overshoot in the angle-of-attack and plunge-acceleration responses. The acceleration response also exhibits some high-frequency oscillation coming from the third aeroelastic mode that contains both pitch and plunge deformations.

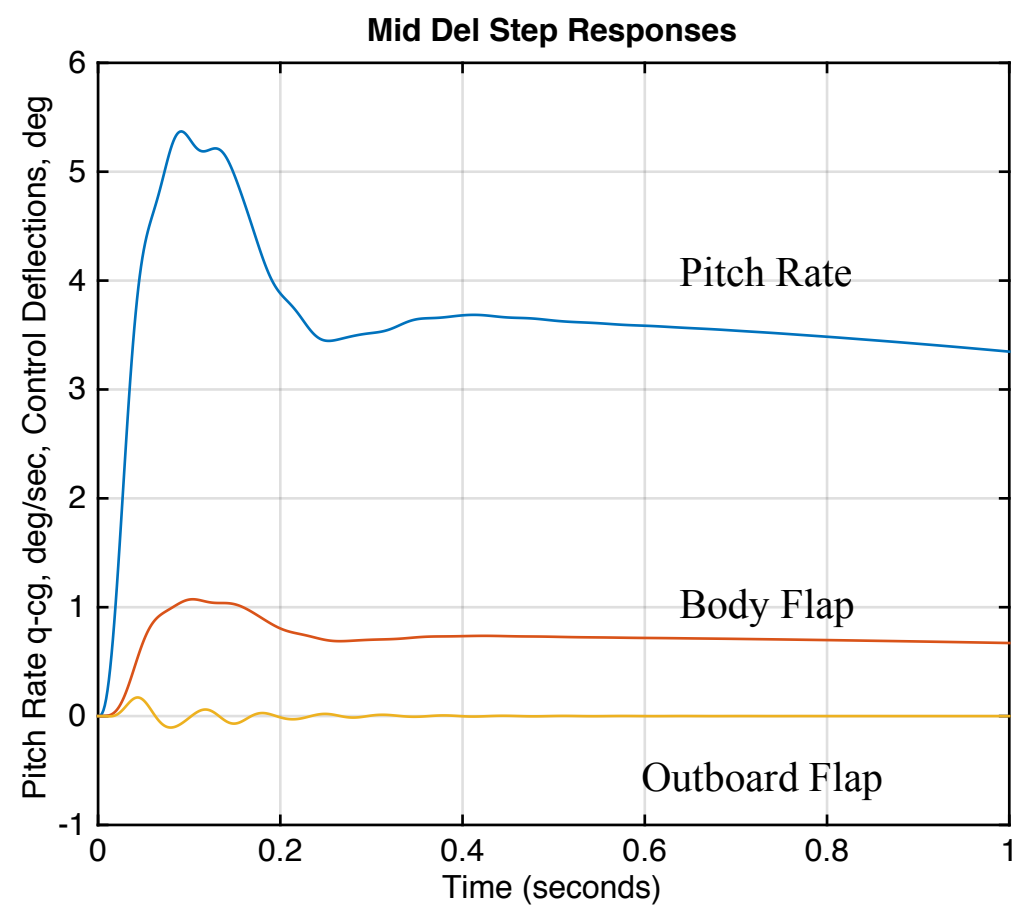

Figure 21a, Pitch-Rate (cg) And Control Surface Step Responses from -1-Deg Del3 


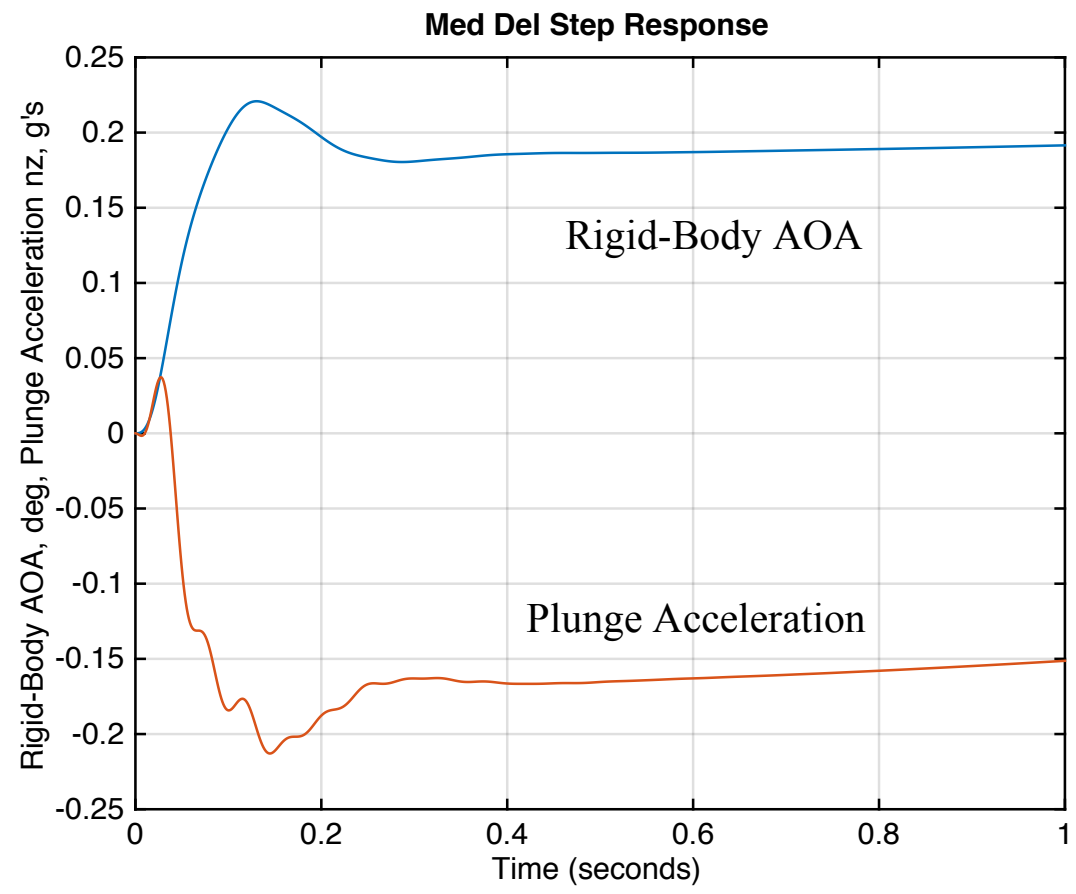

Figure 21b, Mean-Axis AOA and $n_{Z \text {-cg }}$ Step Responses from -1-Deg Del3

This pitch-rate response should be compared to similar responses in Fig. 9, or the pitchrate responses for the rigid and flexible model at the slower flight velocity of $60 \mathrm{fps}$. Except for some small aeroelastic oscillations, the pitch-rate response shown in Fig. 21a is very similar to that for the residualized model in Fig. 9, which is the response of the flexible vehicle with the aeroelastic modes forced to remain in static equilibrium. The similarity between these two sets of pitch-rate responses is also very encouraging.

The body-flap deflection in Fig. 21a mirrors the pitch rate, as expected, and the outboardflap deflection indicates the active damping of the second aeroelastic (torsion) mode. These control-surface deflections do not appear excessive.

\subsection{Additional Filters}

With a constant pitch-rate-feedback gain in the pitch-rate loop the body-flap deflection tracks the center-body pitch rate, as just noted. And in a sustained maneuver this sustained body- 
flap deflection will be such that it counteracts the sustained pitch rate, and require greater deflections of the vehicle's pitch control surface (e.g., elevator). To mitigate this undesirable effect, we may insert a washout filter in the pitch-rate feedback loop, and the BFFsuppression/SAS control law now becomes

$$
\frac{\delta_{B F c}(s)}{q_{c g}(s)}=-0.20\left(\frac{s}{s+1}\right) \mathrm{deg} / \mathrm{deg} / \mathrm{sec}
$$

At low frequencies (below $1 \mathrm{rad} / \mathrm{sec}$ ) the washout filter effectively opens this feedback loop, as desired. And at higher frequencies this filter is essentially unity. Furthermore, since the washout-filter dynamics lie in a frequency rage well below the crossover frequencies of the two control loops, it will have no effect on their stability margins. The main effect of this added filter is indicated in the step responses in Fig 22. These responses are again from a commanded onedegree mid-flap (elevator) input. Comparing these responses with those in Fig. 21a reveals that the vehicle's center-body pitch rate and body-flap deflection slowly decline after 0.4 seconds, as desired. In addition, this filter stabilizes the still lightly damped Phugoid mode.

With regards to the tip-twist loop, accelerometer outputs are being fed back and such outputs tend to be noisy at high frequencies. One would usually not like to drive the actuators with such a signal, so a low-pass filter could be used in the feedback path to alleviate such a situation. In addition, a low-pass filter provides the additional benefit of gain stabilizing highfrequency unmodeled elastic modes. But the corner frequency of the filter would have to be high enough to maintain the loop's performance (i.e, the increase in torsion-mode damping) already limited by the actuator phase loss. So in our case this corner frequency must be higher than we might typically prefer. 


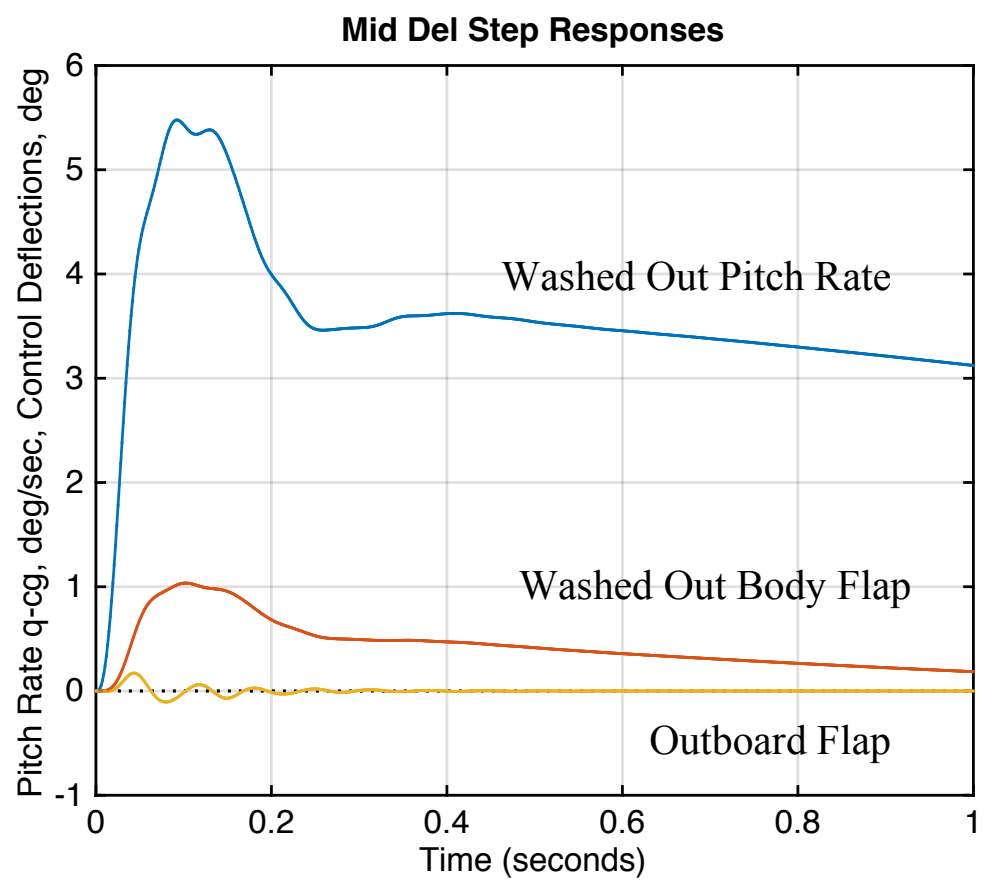

Figure 22, Pitch-Rate (cg) and Controls Step Responses (w. Washout) from 1-Deg Del3

\subsection{Summary of Loop Parameters}

In the $60 \mathrm{kt}$ flight condition it is noted that the BFF condition is more unstable and the second aeroelastic (torsion) mode is now unstable as well. Following the tuning process discussed in Secs. 6.2 and 6.3, and including the filters just discussed, the key control parameters are summarized in Table 9. Note that with the higher instabilities at $60 \mathrm{kt}$, the stability margins are reduced. The closed-loop eigenvalues at $60 \mathrm{kt}$ are given in Table 10.

Table 9, Summary of Controller Results

\begin{tabular}{|c|c|c|c|c|c|c|c|c|}
\hline $\begin{array}{c}\text { Flight } \\
\text { Velocity }\end{array}$ & $\begin{array}{c}\text { BFF } \\
\text { Control } \\
\text { Gain } \\
\mathrm{deg} / \mathrm{deg} / \mathrm{sec}\end{array}$ & $\begin{array}{c}\text { BFF } \\
\text { Gain } \\
\text { Margin } \\
\mathrm{dB}\end{array}$ & $\begin{array}{c}\text { BFF } \\
\text { Phase } \\
\text { Margin } \\
\mathrm{deg}\end{array}$ & $\begin{array}{c}\text { BFF Gain } \\
\text { Crossover } \\
\text { Frequency } \\
\mathrm{rad} / \mathrm{sec}\end{array}$ & $\begin{array}{c}\text { BT } \\
\text { Control } \\
\text { Gain } \\
\mathrm{deg} / \mathrm{deg} / \mathrm{sec}^{2}\end{array}$ & $\begin{array}{c}\text { BT } \\
\text { Gain } \\
\text { Margin } \\
\mathrm{dB}\end{array}$ & $\begin{array}{c}\text { BT } \\
\text { Phase } \\
\text { Margin } \\
\mathrm{deg}\end{array}$ & $\begin{array}{c}\text { BT Gain- } \\
\text { Crossover } \\
\text { Frequency } \\
\mathrm{rad} / \mathrm{sec}\end{array}$ \\
\hline $50 \mathrm{kt}$ & -0.20 & $-20+14$ & $-60+50$ & 45 & $-4 \times 10^{-4}$ & $>20$ & 90 & 90 \\
\hline $60 \mathrm{kt}$ & -0.15 & $-10+14$ & $-60+45$ & 48 & $-5 \times 10^{-4}$ & $-11+12$ & $-85+34$ & 89 \\
\hline
\end{tabular}




Table 10, Closed-Loop Eigenvalues (With Actuators and Filters) - 60 kt
\begin{tabular}{|cccll|}
\hline Eigenvalue, r/s & $\boldsymbol{\varsigma}$ & $\boldsymbol{\omega}, \mathbf{r} / \mathbf{s}$ & Root Locus Branch Identification \\
\hline$-0.0025+/-0.39 \mathrm{i}$ & 0.0064 & 0.392 & Phugoid \\
-1.1 & 1 & 1.1 & Washout-Filter in Pitch Loop \\
$-13.5+/-29.2 \mathrm{i}$ & 0.418 & 32.2 & BFF $\rightarrow$ New Elastic Short Period \\
$-35+/-57.9 \mathrm{i}$ & 0.518 & 67.6 & Former Elastic Short Period \\
$-13.8+/-87.9 \mathrm{i}$ & 0.155 & 89 & Stabilized $2^{\text {nd }}$ Aeroelastic \\
$-74.1+/-53.5 \mathrm{i}$ & 0.811 & 91.4 & Actuator \\
$-63.9+/-79.2 \mathrm{i}$ & 0.628 & 102 & Actuator \\
$-15.9+/-147 \mathrm{i}$ & 0.108 & 148 & $3^{\text {rd }}$ Aeroelastic \\
-1000 & 1 & 1000 & Low-Pass Filter in Tip Loop \\
\hline
\end{tabular}

The negative-one-degree elevator step responses of this closed-loop system are depicted in Figs. 23a and 23b, which show the center-body pitch rate, body-flap and outboard-flap deflections, rigid-body angle of attack, and plunge acceleration at the cg. As in the $50 \mathrm{kt}$ case, the pitch response is dominated by the new elastic-short-period mode, which now has a damping ratio of about 0.42 and an undamped natural frequency of over $30 \mathrm{rad} / \mathrm{sec}$. The washed-out pitch rate and body-flap deflection are evident as well in Fig. 23a. The rigid-body AOA response in Fig. $23 \mathrm{~b}$ is not as clean as that shown in Fig. $22 \mathrm{~b}$ for $50 \mathrm{kt}$. There is more evidence of aeroelastic response in both the pitch rate and rigid-body AOA, but this is not surprising given the higher open-loop BFF instability and the large participation of the elastic degrees of freedom in the new elastic-short-period mode. Again, the control-surface deflections are modest.

\section{Conclusions}

An integrated control law was developed for the stability augmentation (SAS) and active flutter suppression (AFS) of a flexible, flying-wing research drone. A previously developed n-degree-offreedom (NDOF) math model of the longitudinal dynamics was utilized in the control-law synthesis and analysis. Although the vehicle is statically stable in pitch, it is predicted to exhibit 
Submitted to the Journal of Guidance, Control, and Dynamics May 19, 2015

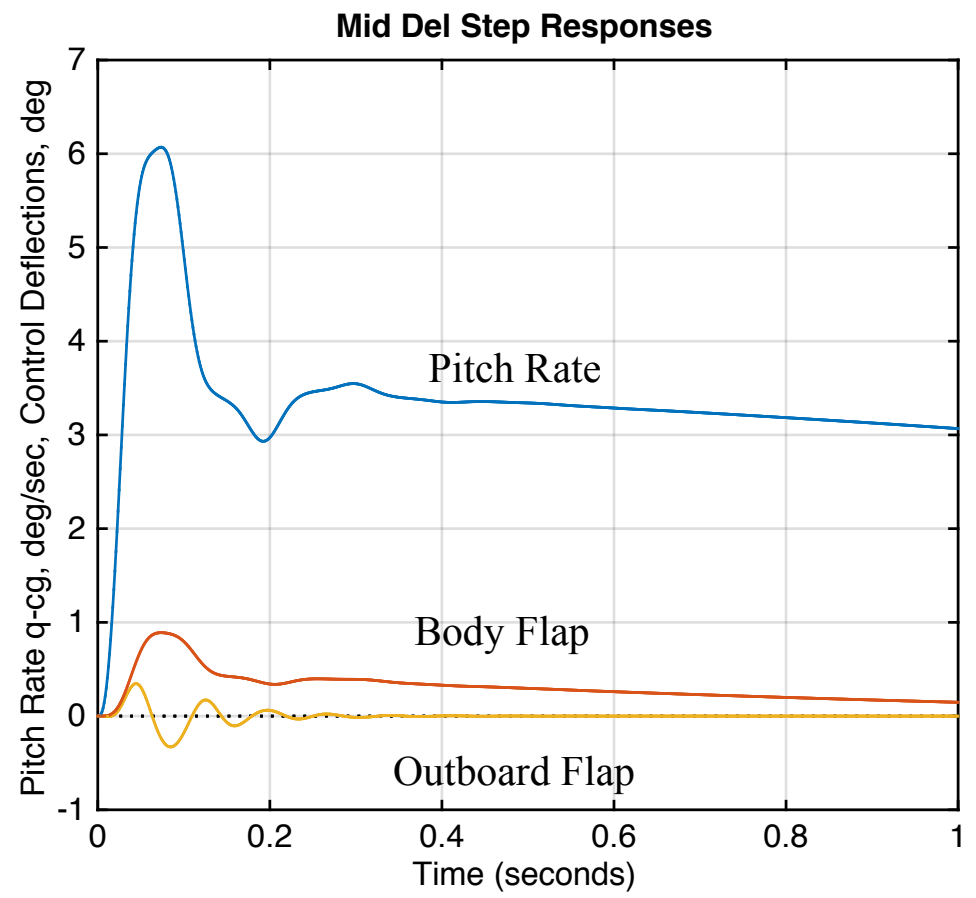

Figure 23a, Pitch-Rate (cg) And Control Surface 1 deg Step Responses - 60 kt,

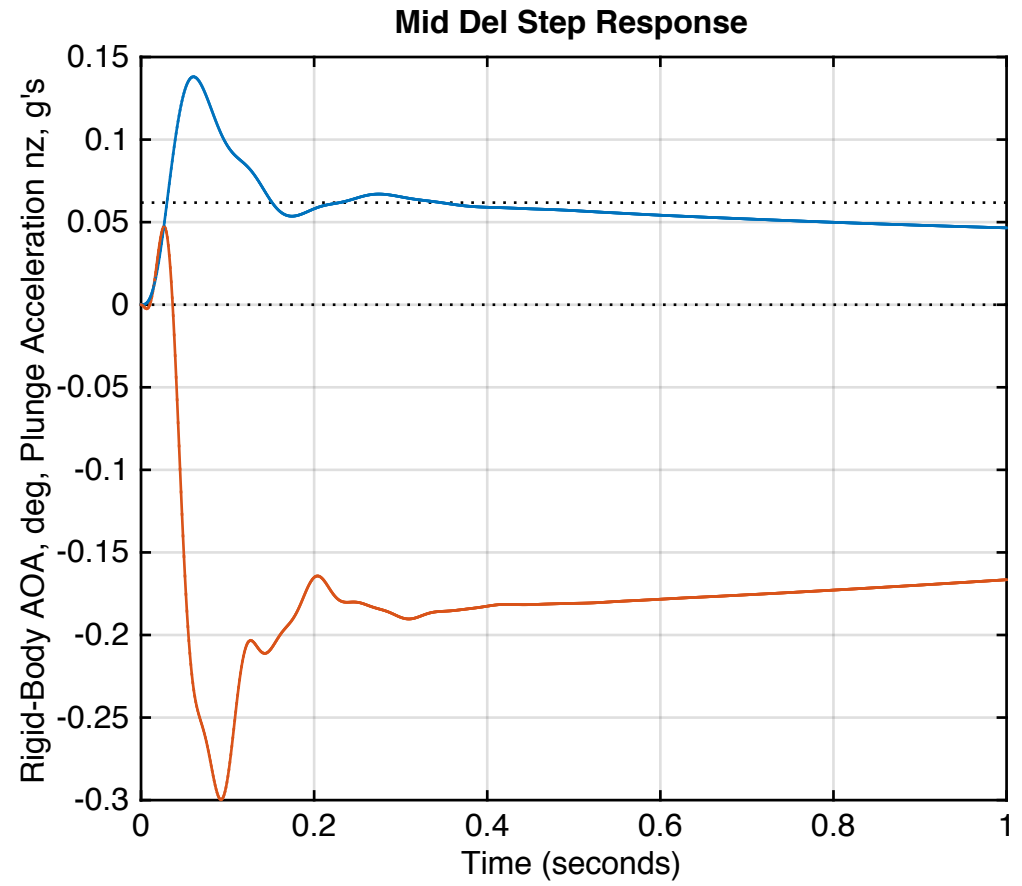

Figure 23b, AOA and Plunge Acceleration (cg) 1-deg Step Responses- 60 kt 
both body-freedom and bending torsion flutter at 47 and $57 \mathrm{kt}$, respectively, and a body-freedom flutter (BFF) speed of $46 \mathrm{kt}$ was observed in flight tests.

BFF involves the aeroelastic interactions between the rigid-body and elastic degrees of freedom; hence the control laws must simultaneously address both rigid-body stability augmentation and flutter suppression. The overall approach taken in the control-law synthesis was motivated by the concept known as Identically Located Force and Acceleration (ILAF. This concept is powerful since it does not rely on precise knowledge of the structural mode shapes, which are difficult to predict accurately and change with changes in mass and stiffness properties of the structure.

The crux of the flutter-suppression problem was shown to be the interactions between the vehicle's rigid-body pitching and the first symmetric bending/torsion (pitch) mode, as well as the interactions between these modes and the second symmetric torsion mode. Based on these facts and consideration of the vibration mode shapes and available control surfaces, ILAF and conventional SAS techniques suggested two loop closures - feedback of center-body pitch rate to the body flaps and feedback of blended wing-tip accelerometers (wing torsion) to the outboard wing control surfaces. Analysis was performed at two flight conditions, first at a $50 \mathrm{kt}$ flight velocity, below the second flutter speed so only the BFF condition was unstable, and a second at $60 \mathrm{kt}$ that exhibits two flutter conditions. It was confirmed that these two feedback loops robustly stabilized both flutter conditions, and they were also shown to appropriate affect the modal characteristics of the longitudinal dynamics, leading to quite reasonable vehicle attitude response. A washout filter was also employed to tailor the low-frequency response and stabilize the Phugoid mode, and the control deflections do not appear excessive. The critical factors limiting 
the performance of the feedback system have been identified to be the bandwidth of the surface actuators and the pitch effectiveness of the body flaps.

\section{Acknowledgement}

The author would like to remember our friend and colleague Dr. Gary Balas, former head of the Department of Aerospace Engineering and Mechanics at the UMN, and express his thanks for the opportunity to collaborate on this research. This work was supported under NASA Cooperative Agreement No. NNX14AL63A, with the University of Minnesota the prime contractor. Mr. John Bosworth and Dr. Dan Moerder of NASA, have served as technical monitors. This support is gratefully appreciated.

\section{References}

1. Burnett, Edward L., et al, “ NDOF Simulation Model for Flight Control Development with Flight Test Correlation,” Lockheed Martin Aeronautics Co., AIAA Modeling and Simulation Technologies Conf., Paper No. 2010-7780, Toronto, August 2-5, 2010.

2. Kotikalpudi, Aditya, et al, "Swing Tests for Estimation of Moments of Inertia," Unpublished notes, University of Minnesota Dept. of Aerospace Engineering and Mechanics, 2013.

3. Schmidt, D.K. "MATLAB ${ }^{\circledR}$-Based Flight-Dynamics and Flutter Analysis of a Flexible Flying-Wing Research Drone," Working Paper prepared for the UMN NASA PAAW Project, November, 2014. Submitted to the Journal of Aircraft.

4. Schmidt, David K., Modern Flight Dynamics, McGraw-Hill, 2012.

5. Bisplinghoff, R. L., Ashley, H., Principles of Aeroelasticity. John Wiley \& Sons, Inc., 1962.

6. Morino, Claudia P., "Finite-Element Structural Analysis of a Small Flexible Aircraft," Unpublished notes, University of Minnesota Dept of Aerospace Engineering and Mechanics, 2014.

7. Moreno, Claudia P., et al, "Structural Model Identification of a Small Flexible Aircraft," presented at the American Control Conference, Portland, OR, June 2014. 
Submitted to the Journal of Guidance, Control, and Dynamics May 19, 2015

8. Wykes, Borland, Klepl, and MacMiller, "Design and Development of a Structural Mode Control System," NASA Contractor Report CR-143846, Prepared by Rockwell International, Inc., October 1977. 